NISSUNA UMANA INVESTIGAZIONE SI PUO DIMANDARE VERA SCIENZIA S'ESSA NON PASSA PER LE MATEMATICHE DIMOSTRAZIONI LEONARDO DA VINCI

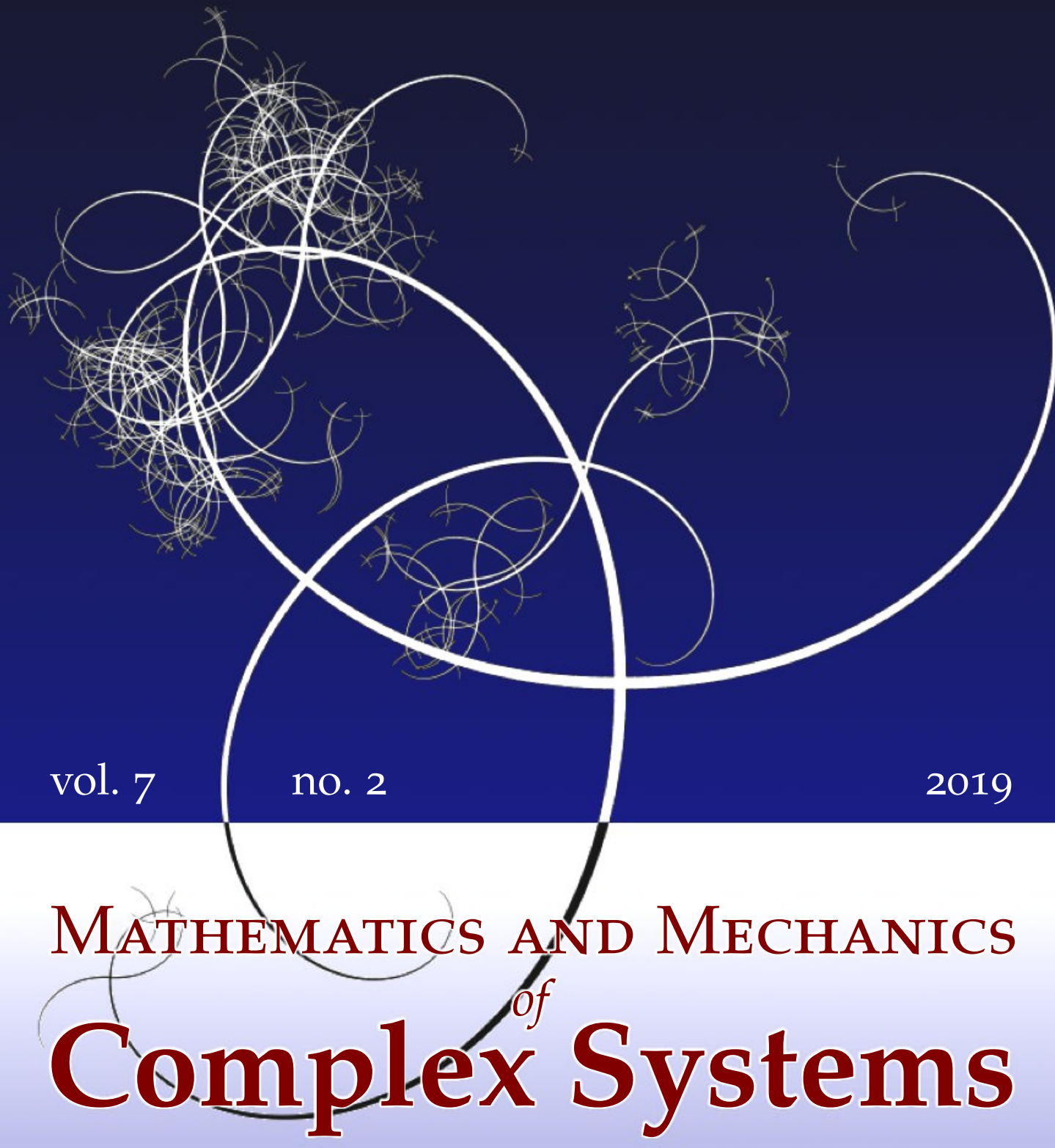

Eduard Penner, Ismail Caylak, Alex Dridger and Rolf Mahnken

A POLYNOMIAL CHAOS EXPANDED

HYBRID FUZZY-STOCHASTIC MODEL

FOR TRANSVERSELY FIBER REINFORCED PLASTICS 


\title{
A POLYNOMIAL CHAOS EXPANDED HYBRID FUZZY-STOCHASTIC MODEL FOR TRANSVERSELY FIBER REINFORCED PLASTICS
}

\author{
Eduard Penner, Ismail Caylak, Alex Dridger and Rolf Mahnken
}

\begin{abstract}
This work is focused on polymorphic uncertainties in the framework of constitutive modeling for transversely isotropic materials. To this end, we propose a hybrid fuzzy-stochastic model, where the stochastic part accounting for aleatory uncertainties of material parameters is expanded with the multivariate polynomial chaos expansion. In order to account for epistemic uncertainties, polynomial chaos coefficients are treated as fuzzy variables. The underlying minimum and maximum optimization problem for the fuzzy analysis is approximated by $\alpha$-level discretization, resulting in a separation of minimum and maximum problems. To become more universal, so-called quantities of interest are employed, which allow a general formulation for the target problem. Numerical examples with fuzzy, fuzzy-stochastic, and hybrid fuzzy-stochastic input demonstrate the versatility of the proposed formulation.
\end{abstract}

\section{Introduction}

A fundamental data uncertainty of different types underlies most materials in engineering science. Possible examples are variations in the manufacturing process, where composites are typical materials, measurement errors, and missing or incomplete information. In order to improve the credibility of mathematical models in engineering science, uncertainties have to be taken into account, where two categories are distinguished: aleatory and epistemic; see, e.g., [Sullivan 2015].

Aleatory uncertainties refer to variability as a consequence of, e.g., fluctuations through time, variation across space, or manufacturing differences. This type of uncertainty is irreducible and can be treated with a stochastic analysis. An aleatory uncertain problem of a mechanical system can be modeled by stochastic partial differential equations (SPDEs), where the system response may be described by a distribution with statistical moments. The solution of these SPDEs can be obtained

\section{Communicated by Paul Steinmann.}

MSC2010: 60A86.

Keywords: polymorphic uncertainty, fuzzy, stochastic, fuzzy-stochastic, polynomial chaos expansion, fiber reinforced plastics, transversely isotropic materials, imprecise probability, epistemic, aleatory. 
numerically using a stochastic simulation, where the Monte Carlo (MC) method [Caflisch 1998; Hurtado and Barbat 1998] is widely used. Alternatively, spectral stochastic surrogate models, e.g., polynomial chaos expansion (PCE), are used in order to reduce the computational effort. Corresponding research areas are: linear elasticity of solids and mechanics [Ghanem and Spanos 1991], plasticity of solids and mechanics [Anders and Hori 1999; Rosić 2013], large deformations [Acharjee and Zabaras 2006; Acharjee 2006; Caylak et al. 2018], fluid flow [Le Maître et al. 2001; 2002], flow-structure interactions [Xiu and Karniadakis 2002; Xiu et al. 2001], and linear convection problems [Jardak et al. 2002].

Contrary to aleatory uncertainties, epistemic uncertainties refer to subjectivity as a consequence of, e.g., incomplete scientific understanding or lack of measurements, which indicate a possible value range rather than a probability function. In addition, epistemic uncertainties are reducible by empirical effort, e.g., investing more in measurements. Methodologies for the modeling of epistemic uncertainties are, e.g., interval analysis and, increasingly applied over the last years, fuzzy analysis, which represents indistinct boundaries [Zadeh 1965]. In order to perform mathematical operations with fuzzy sets, the so-called $\alpha$-level discretization method is applied. Here, the fuzzy response at each selected $\alpha$-level is obtained by solving a minimum-maximum problem of a quantity of interest (QoI). In [Mahnken 2017] QoIs are employed within a variational formulation for fuzzy analysis in continuum mechanics.

A realistic modeling of uncertainties requires a combination of different uncertainty types. Following [Graf et al. 2015], this is referred to as polymorphic uncertainties. Corresponding models are: Dempster-Shafer evidence theory [Dempster 1967], coherent lower prevision theory [Walley 1991], possibility theory [Dubois and Prade 2012], probability box (P-box) theory [Ferson et al. 2003], and fuzzy probability theory [Gudder 1998; Beer 2009] or fuzzy-randomness [Möller and Beer 2004]. Recently published works using fuzzy-randomness are in the field of civil engineering [Reuter et al. 2012], fuzzy-random dynamical structural analysis [Graf et al. 2015], failure probability evaluation [Jahani et al. 2014], and fuzzystochastic partial differential equations [Motamed 2017].

Note that the QoI in the publications listed in the previous paragraph may be a fuzzy-stochastic random variable. The numerical computation of the stochastic part is realized by sampling methods which require a large number of samples. From the received samples, different statistical moments can be computed to solve the minimum-maximum problem of the QoI at each $\alpha$-level. Thus, at each optimization step, statistical moments must be determined, which lead to high computational costs. In order to reduce this effort, we introduce a novel hybrid fuzzy-stochastic model based on the polynomial chaos expansion. In particular the following aspects are investigated: 
- The stochastic part of the hybrid polymorphic model is expanded with the PCE.

- For the fuzzy part of the hybrid polymorphic model, the polynomial chaos (PC) coefficients are treated as fuzzy variables.

- At each optimization step, the statistical moment only depends on deterministic PC coefficients.

- Experimental investigation and uncertainty quantification of transversely fiber reinforced plastics are carried out.

This paper is structured as follows. Section 2 provides the stochastic formulation for the state and target problem and their discretizations with the PCE. In Section 3 the hybrid fuzzy-stochastic formulation and the computational scheme for the numerical implementation with polymorphic uncertainties are proposed. Section 4 incorporates the experimental investigation and the parameter identification for fiber reinforced plastics (FRP). Finally, in Section 5 the representative numerical examples demonstrate the versatility of the proposed model considering different types of uncertainties.

\section{Stochastic analysis}

2.1. Constitutive stochastic state problem. To set the stage for a hybrid fuzzystochastic model accounting for polymorphic uncertainties, we introduce a design vector

$$
\left[s_{1}, \ldots, s_{n_{s}}\right]^{T}=\underline{s} \in \mathscr{S}
$$

within a design space $\mathscr{S} \subset \mathbb{R}^{n_{s}}$ of $n_{s}$ design variables. In general it may represent several influences on a structure, such as material properties, loading parameters, geometric properties, and boundary conditions. In this section, we assume the design space $\mathscr{Y} \subset \mathbb{R}^{n_{s}}$ in (1) is a precise set or a fundamental set of ordered pairs. The aleatory uncertainties are modeled by stochastic random variables. In this context the probability space is denoted by $(\Omega, \Sigma, \mathbb{P})$, where $\Omega$ is the set of elementary events, $\Sigma$ is the $\sigma$-algebra, and $\mathbb{P}$ is the probability measure. Moreover, we let $\omega$ be an element of $\Omega$.

Typically, constitutive modeling in mechanics is based on stress-strain relations. To this end, we introduce a stress-strain space $M \subset \mathscr{E}^{3} \times \mathscr{E}^{3}$, where $\mathscr{E}^{3}$ denotes the Euclidean space. An uncertain functional for a strain tensor $\varepsilon$ on the design space $\mathscr{S}$ and on the probability space $\Omega$ may be interpreted as a random variable $\boldsymbol{\varepsilon}(\cdot, \underline{s}): \Omega \rightarrow \mathcal{M}$ indexed by $\underline{\underline{s}} \in \mathscr{Y}$ on the design space $\mathscr{Y}$, where for any elementary event $\omega$ yields a realization $\boldsymbol{\varepsilon}(\omega, \cdot): \mathscr{S} \rightarrow \mathcal{M}$; see, e.g., [Wang and Zhang 1992]. 


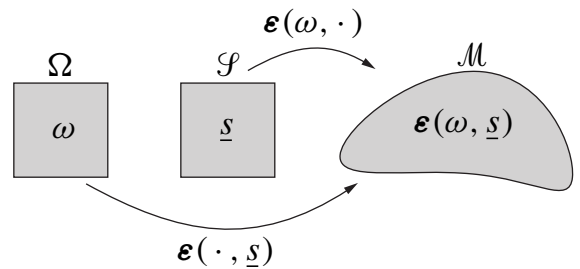

Figure 1. Mapping from stochastic and design space to strain space.

In both interpretations, an uncertain functional is a measurable function

$$
\boldsymbol{\varepsilon}(\omega, \underline{s}):=\left\{\begin{array}{l}
\Omega \times \mathscr{Y} \rightarrow M \subset \mathscr{E}^{3} \times \mathscr{E}^{3}, \\
(\omega, \underline{s}) \mapsto \boldsymbol{\varepsilon}(\omega, \underline{s}) .
\end{array}\right.
$$

The mapping $\varepsilon(\cdot, \cdot)$ from stochastic and design space to strain space is visualized in Figure 1, where for simplicity time dependence, explicit probabilistic dependence, and explicit design dependence are not considered in the illustration.

Based on the stochastic strain-driven mapping equation (2), a stochastic linear elastic constitutive problem can be formulated as

$$
\begin{array}{lll}
\text { 1. (constitutive equation) } & \boldsymbol{\sigma}=\mathbb{C}(\underline{\kappa}(\omega, \underline{s})): \boldsymbol{\varepsilon}(\underline{\kappa}(\omega, \underline{s})) \in \mathcal{M}, \\
\text { 2. (stress constraint) } & \boldsymbol{\sigma}=\overline{\boldsymbol{\sigma}}(\omega, \underline{s}) & \in M_{\sigma} \subset \mathcal{M}, \\
\text { 3. (strain constraint) } & \boldsymbol{\varepsilon}=\overline{\boldsymbol{\varepsilon}}(\omega, \underline{s}) & \in M_{\varepsilon} \subset \mathcal{M},
\end{array}
$$

where

$$
\underline{\kappa}(\omega, \underline{s})=\left[\kappa_{1}(\omega, \underline{s}), \ldots, \kappa_{i}(\omega, \underline{s}), \ldots, \kappa_{n_{m}}(\omega, \underline{s})\right]^{T}
$$

is the material parameter vector of $n_{m}$ polymorphic uncertain material parameters. In addition, in (3) $)_{1}$ the symmetric Cauchy stress tensor $\sigma$ and the polymorphic uncertain elasticity material tensor $\mathbb{C}(\underline{\kappa}(\omega, \underline{s}))$ are used. Stress and strain constraints in (3) $)_{2}$ and (3) 3 are formulated on the spaces $\mu_{\sigma}$ and $\mu_{\varepsilon}$, where $\mu_{\sigma} \cup M_{\varepsilon}=\mathcal{M}$ and $\mu_{\sigma} \cap M_{\varepsilon}=\varnothing$ hold, and defined by prescribed stresses $\bar{\sigma}(\omega, \underline{s})$ and strains $\overline{\boldsymbol{\varepsilon}}(\omega, \underline{s})$, respectively. In the sequel, we assume that $\underline{\kappa}(\omega, \underline{s})$ is the only uncertain input variable that depends on the aleatory elementary event $\omega$ and on the design variables $\underline{s}$. We exploit a strain based method and formulate

$$
\begin{aligned}
& \text { the stochastic state problem: } \\
& \text { for given } \underline{s} \in \mathscr{Y} \text { and } \omega \in \Omega \text { find } \boldsymbol{\varepsilon}(\underline{\kappa}(\omega, \underline{s})) \text { such that } \\
& \qquad \boldsymbol{r}(\underline{\kappa}(\omega, \underline{s})):=\underbrace{\mathbb{C}(\underline{\kappa}(\omega, \underline{s})): \boldsymbol{\varepsilon}(\underline{\kappa}(\omega, \underline{s}))}_{\sigma(\underline{\varepsilon}(\underline{\kappa}(\omega, \underline{s})))}-\overline{\boldsymbol{\sigma}}=\mathbf{0},
\end{aligned}
$$

where $\boldsymbol{r}(\underline{\kappa}(\omega, \underline{s}))$ has the interpretation of a stochastic residual for a strain-driven algorithm. 
2.2. Stochastic quantity of interest and target problem. In the subsequent exposition we do not merely want to characterize the design properties of the random strain tensor $\boldsymbol{\varepsilon}(\underline{\kappa}(\omega, \underline{s}))$ satisfying the stochastic state equation (5). Instead, we are more interested in the stochastic design analysis of a physical event or a feature of a structure that depends upon $\boldsymbol{\varepsilon}$, where quantities of interest (QoIs) are characterized by functionals $Q(\boldsymbol{\varepsilon}(\underline{\kappa}(\omega, \underline{s})))$ of the solutions $\boldsymbol{\varepsilon}(\underline{\kappa}(\omega, \underline{s}))$ to (5). A possible realization of the QoI is

$$
Q(\varepsilon(\underline{\kappa}(\omega, \underline{s})))=\sigma(\varepsilon(\underline{\kappa}(\omega, \underline{s}))): \sigma(\varepsilon(\underline{\kappa}(\omega, \underline{s}))) .
$$

Note that the QoI may be a stochastic random variable $Q(\underline{\kappa}(\omega, \underline{s}))$ that depends on $\omega$ and $\underline{s}$. Therefore, it is common to generate a relatively large number $n_{\mathrm{MC}}$ of samples $Q_{j}(\underline{s}), j=1, \ldots, n_{\mathrm{MC}}$, for $Q(\underline{\kappa}(\omega, \underline{s}))$, as, e.g., in the Monte Carlo (MC) simulation, based on its stochastic distribution described by a probability density function and to evaluate a QoI based on the computed results of all samples $Q_{j}(\underline{s})$, $j=1, \ldots, n_{\mathrm{MC}}$. From the received samples, different deterministic values can be computed to evaluate a so-called surrogate $Q o I Q^{\omega}$. The upper index $\omega$ indicates that $Q^{\omega}$ is a surrogate QoI with respect to a QoI $Q$. Possible realizations for the surrogate $\mathrm{QoI} Q^{\omega}$ of the state problem in (5) are:

- expectation value (or sample mean)

$$
Q^{\omega}=\mathbb{E}[Q(\boldsymbol{\varepsilon}(\underline{\kappa}(\omega, \underline{s})))] \approx \frac{1}{n_{\mathrm{MC}}} \sum_{j=1}^{n_{\mathrm{MC}}} Q_{j},
$$

- variance (or adjusted sample variance)

$$
Q^{\omega}=\operatorname{var}[Q(\boldsymbol{\varepsilon}(\underline{\kappa}(\omega, \underline{s})))] \approx \frac{1}{n_{\mathrm{MC}}-1} \sum_{j=1}^{n_{\mathrm{MC}}}\left(Q_{j}-\mathbb{E}[Q]\right)^{2},
$$

- square norm in $L_{2}(\Omega, \Sigma, \mathbb{P})$ or second moment, also called stochastic norm [Le Maître and Knio 2010]

$$
Q^{\omega}=\mathbb{E}\left[Q^{2}(\boldsymbol{\varepsilon}(\underline{\kappa}(\omega, \underline{s})))\right] \approx \frac{1}{n_{\mathrm{MC}}} \sum_{j=1}^{n_{\mathrm{MC}}} Q_{j}^{2},
$$

- functional dependencies of stochastic and design variables

$$
Q^{\omega}=Q^{\omega}(\underline{\kappa}(\omega, \underline{s})),
$$

whereby surrogate QoIs (7)-(9) are based on sample empirical moments. The adjusted sample variance in (8) refers to the fact that the sum of squared deviations is divided by $n_{\mathrm{MC}}-1$ rather than by $n_{\mathrm{MC}}$. With a specific choice of a surrogate 
QoI $Q^{\omega}$, for example according to (7)-(10), we formulate

$$
\begin{aligned}
& \text { the stochastic target problem: } \\
& \text { for given } \underline{s} \in \mathscr{S} \text { and } \omega \in \Omega \text { find } z:=Q^{\omega}(\underline{s}, \boldsymbol{\varepsilon}(\underline{\kappa}(\omega, \underline{s}))) \text {. }
\end{aligned}
$$

Note that, due to the dependency $\boldsymbol{\varepsilon}(\underline{\kappa}(\omega, \underline{s}))$, solution of the target problem in (11) involves solution of the state problem in (5).

2.3. Discretization by polynomial chaos expansion. As mentioned before in Section 2.2, the MC simulation, based on a relatively large number of samples, could be used for numerical evaluation of the QoI in (6). However, if only individual empirical moments in (7)-(9) are sought, a discrete surrogate model, e.g., in terms of the polynomial chaos expansion (PCE) [Ghanem and Spanos 1991; Caylak et al. 2018] can be used in order to reduce the numerical effort. This expansion involves a basis of known random functions with deterministic PC coefficients. Therefore, stochastic variables $\underline{\kappa}(\omega, \underline{s})$ may be represented with the PCE

$$
\underline{\kappa}(\omega, \underline{s}) \approx \sum_{l=0}^{n_{P}} \underline{\hat{\kappa}}_{l}(\underline{s}) \Psi_{l}(\underline{\theta}(\omega))=\underline{\hat{\kappa}}_{0}(\underline{s})+\sum_{l=1}^{n_{P}} \underline{\hat{\kappa}}_{l}\left(\underline{s}_{)} \Psi_{l}(\underline{\theta}(\omega)),\right.
$$

where $\underline{\hat{\kappa}}_{l}(\underline{s})$ are $n_{m} \times 1$ PC coefficient vectors, $n_{P}+1$ is the number of the accompanying PC terms, and $\Psi_{l}(\underline{\theta}(\omega))$ are PC basis functions described by multivariate single-index polynomials with uncorrelated standard distributed random variables $\underline{\theta}$.

In this paper Hermite polynomials with standard normal distributed random variables $\underline{\theta}=\left[\theta_{1}, \ldots, \theta_{n_{m}}\right]$ are chosen. Table 1 provides a single-index representation with a polynomial order $p=1$ for $n_{m}=5$ stochastic parameters. For a more detailed description, we refer to [Keese 2004]. The 0-th PC coefficient in (12) represents the expectation value $\underline{\hat{\kappa}}_{0}(\underline{s})=\mathbb{E}[(\underline{\kappa}(\omega, \underline{s}))]$. With (12) the $i$-th material parameter

\begin{tabular}{|ccl|}
\hline order $p$ & $l$ & $\Psi_{l}$ \\
\hline 0 & 0 & $\Psi_{0}=1$ \\
1 & 1 & $\Psi_{1}=\theta_{1}$ \\
& 2 & $\Psi_{2}=\theta_{2}$ \\
& 3 & $\Psi_{3}=\theta_{3}$ \\
& 4 & $\Psi_{4}=\theta_{4}$ \\
& 5 & $\Psi_{5}=\theta_{5}$ \\
\hline
\end{tabular}

Table 1. Single-index representation of the multivariate polynomials for $n_{m}=5$ stochastic parameters and polynomial order $p=1$, which leads to $n_{P}=5$. 
can be expanded as

$$
\kappa_{i}(\omega, \underline{s}) \approx \sum_{l=0}^{n_{P}} \hat{\kappa}_{i l}(\underline{s}) \Psi_{l}(\underline{\theta}(\omega))=\hat{\kappa}_{i 0}(\underline{s})+\sum_{l=1}^{n_{P}} \hat{\kappa}_{i l}(\underline{s}) \Psi_{l}(\underline{\theta}(\omega)), \quad i=1, \ldots, n_{m} .
$$

Note that in the deterministic case of the $i$-th material parameter with $n_{P}=0$ and the independence on $\underline{s}$, (13) renders $\kappa_{i}=\hat{\kappa}_{i 0}$. Similarly, a nonstochastic $i$-th material parameter with $n_{P}=0$ is given by $\kappa_{i}(\underline{s})=\hat{\kappa}_{i 0}(\underline{s})$.

Inserting (12) into the stochastic state problem of (5) and applying the Galerkin projection [Ghanem and Spanos 1991] renders

\section{the discrete stochastic state problem:}

for given $\underline{s} \in \mathscr{Y}$ and $\omega \in \Omega$ find $\hat{\boldsymbol{\varepsilon}}_{m}(\underline{s}, \underline{\hat{\kappa}})$ such that

$$
\begin{aligned}
\boldsymbol{r}_{k}(\underline{\kappa}(\omega, \underline{s})): & =\sum_{l=0}^{n_{P}} \sum_{m=0}^{n_{P}} c_{k l m}(\omega) \widehat{\mathbb{C}}_{l}(\underline{s}, \underline{\hat{\kappa}}): \hat{\boldsymbol{\varepsilon}}_{m}(\underline{s}, \underline{\hat{\kappa}})-\overline{\boldsymbol{\sigma}}_{k} \\
& =0 \quad \text { for all } k \in\left\{0, \ldots, n_{P}\right\},
\end{aligned}
$$

where

$$
\begin{aligned}
\text { 1. } & c_{k l m}(\omega) & =\mathbb{E}\left[\Psi_{k} \Psi_{l} \Psi_{m}\right], \\
\text { 2. } & \overline{\boldsymbol{\sigma}}_{k} & =\mathbb{E}\left[\overline{\bar{\sigma}} \Psi_{k}\right], \\
\text { 3. } & \boldsymbol{r}_{k}(\underline{\kappa}(\omega, \underline{s})) & =\mathbb{E}\left[\boldsymbol{r}(\underline{\kappa}(\omega, \underline{s})) \Psi_{k}\right] .
\end{aligned}
$$

In (14) $\underline{\hat{\kappa}}(\underline{s})$ describes the $n_{m} \times\left(n_{P}+1\right)$ matrix of PC coefficients

$$
\underline{\hat{\kappa}}(\underline{s})=\left[\underline{\hat{\kappa}}_{0}(\underline{s}), \ldots, \underline{\hat{\kappa}}_{n_{P}}(\underline{s})\right]=\left[\begin{array}{ccc}
\hat{\kappa}_{10}(\underline{s}) & \ldots & \hat{\kappa}_{1 n_{P}}(\underline{s}) \\
\vdots & \ddots & \vdots \\
\hat{\kappa}_{n_{m} 0}(\underline{s}) & \ldots & \hat{\kappa}_{n_{m}\left(n_{P}\right)}(\underline{s})
\end{array}\right] \in \mathbb{R}^{n_{m} \times\left(n_{P}+1\right)} .
$$

With the above preliminaries at hand, the PCE based discrete surrogate QoI $Q^{\omega}$ for an arbitrary QoI $Q$ can be formulated using the PCE in (12). The PCE based discrete QoI is

$$
Q(\underline{\kappa}(\omega, \underline{s}))=\sum_{k=0}^{n_{P}} \widehat{Q}_{k}(\underline{s}, \underline{\hat{\kappa}}) \Psi_{k}(\underline{\theta}(\omega)),
$$

where $\widehat{Q}_{k}(\underline{s}, \underline{\hat{\kappa}})$ are the corresponding PC coefficients. For a specific case, inserting the QoI in (6) into the PC expanded QoI in (17) renders the PC coefficients as

$$
\widehat{Q}_{k}=\frac{1}{d_{k}} \sum_{l=0}^{n_{P}} \sum_{m=0}^{n_{P}} c_{k l m} \hat{\sigma}_{l} \hat{\sigma}_{m} \quad \text { for all } k \in\left\{0, \ldots, n_{P}\right\},
$$


where $c_{k l m}$ is given in $(15)_{1}$ and

$$
d_{k}=c_{k k 0}=\mathbb{E}\left[\Psi_{k}^{2}\right] \text { for all } k \in\left\{0, \ldots, n_{P}\right\} .
$$

With the PCE based discrete QoI in (17) the discrete surrogate QoIs of (7)-(9) become

$$
\begin{aligned}
& Q^{\omega}=\mathbb{E}[Q(\underline{\kappa}(\omega, \underline{s}))]=\widehat{Q}_{0}(\underline{s}, \underline{\hat{\kappa}}), \\
& Q^{\omega}=\operatorname{var}[Q(\underline{\kappa}(\omega, \underline{s}))]=\sum_{k=1}^{n_{P}} d_{k} \widehat{Q}_{k}^{2}(\underline{s}, \underline{\hat{\kappa}}), \\
& Q^{\omega}=\mathbb{E}\left[Q^{2}(\underline{\kappa}(\omega, \underline{s}))\right]=\sum_{k=0}^{n_{P}} d_{k} \widehat{Q}_{k}^{2}(\underline{s}, \underline{\hat{\kappa}}) ;
\end{aligned}
$$

see, e.g., [Le Maître and Knio 2010]. In (20)-(22) it can be seen that the surrogate QoIs of random variables depend only on the design variables $\underline{s}$ in (1), the deterministic PC coefficients $\underline{\hat{\kappa}}(\underline{s})$ in (16), and the expectation values in (19).

With a specific choice of a discrete surrogate QoI $Q^{\omega}$, for example according to (20)-(22), we formulate

$$
\begin{aligned}
& \text { the discrete stochastic target problem: } \\
& \text { for given } \underline{s} \in \mathscr{Y} \text { and } \omega \in \Omega \text { find } z:=Q^{\omega}(\underline{s}, \underline{\hat{\boldsymbol{\varepsilon}}}(\underline{s}, \underline{\hat{\kappa}}), \underline{d}) \text {, }
\end{aligned}
$$

where $\underline{\hat{\boldsymbol{\varepsilon}}}(\underline{s}, \underline{\hat{\kappa}})=\left[\hat{\boldsymbol{\varepsilon}}_{0}(\underline{s}, \underline{\hat{\kappa}}), \ldots, \hat{\boldsymbol{\varepsilon}}_{n_{P}}(\underline{s}, \underline{\hat{\kappa}})\right]^{T}$ and $\underline{d}=\left[d_{0}, \ldots, d_{n_{P}}\right]^{T}$. Note that, due to the dependency $\underline{\hat{\varepsilon}}(\underline{s}, \underline{\hat{\kappa}})$, solution of the discrete target problem in (23) involves solution of the discrete state problem in (14).

2.4. Example. In order to demonstrate the concept of a surrogate QoI in linear isotropic elasticity we consider a uniaxial strain-driven loading case with $\varepsilon=0.1$. Furthermore, a PC expanded Young's modulus $E(\omega)$ is assumed as a normal distributed random variable according to (13) with $n_{m}=1$ and $n_{P}=1$ :

$$
E(\omega)=\widehat{E}_{0}+\widehat{E}_{1} \theta(\omega)=80 \mathrm{GPa}+5 \mathrm{GPa} \theta(\omega) .
$$

In Figure 2, left, the distribution of the random variable in (24) is illustrated as a solid line, where in addition the histogram represents Young's modulus $E(\omega)$ using $n_{\mathrm{MC}}=10^{8}$ Monte Carlo samples. From the solution of the discrete stochastic state problem in (14), we obtain the stress random variable $\sigma(\omega)$ as

$$
\sigma(\omega)=\hat{\sigma}_{0}+\hat{\sigma}_{1} \theta(\omega)=\widehat{E}_{0} \varepsilon+\widehat{E}_{1} \varepsilon \theta(\omega)=8 \mathrm{GPa}+0.5 \mathrm{GPa} \theta(\omega) .
$$

The stress PC coefficients in (25) are used to calculate the QoI PC coefficients with $n_{P}=1$ according to (18), where $c_{k l m}$ and $d_{k}$ in (15) 1 and (19), respectively, for 

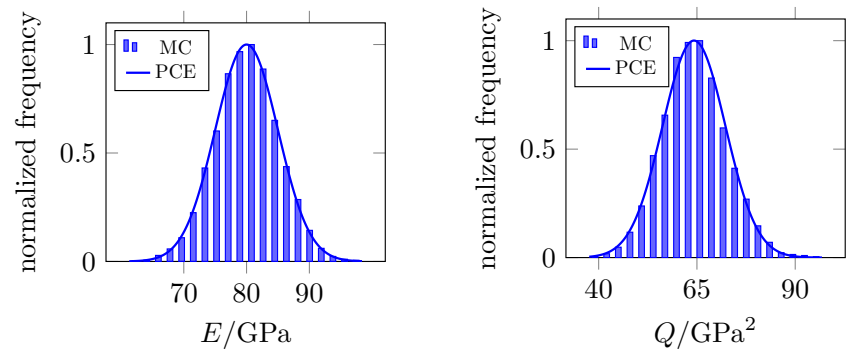

Figure 2. PCE and MC distribution of Young's modulus $E(\omega)$ (left) and QoI $Q(\omega)$ (right) of (24) and (27), respectively.

\begin{tabular}{|c|c|c|c|c|}
\hline$k$ & $l$ & $m$ & $c_{k l m}$ & $d_{k}$ \\
\hline \multirow{4}{*}{0} & 0 & 0 & 1 & \multirow{4}{*}{1} \\
\hline & 0 & 1 & 0 & \\
\hline & 1 & 0 & 0 & \\
\hline & 1 & 1 & 1 & \\
\hline \multirow{4}{*}{1} & 0 & 0 & 0 & \multirow{4}{*}{1} \\
\hline & 0 & 1 & 1 & \\
\hline & 1 & 0 & 1 & \\
\hline & 1 & 1 & 0 & \\
\hline
\end{tabular}

Table 2. Values of $c_{k l m}$ and $d_{k}$ for a stochastic dimension $n_{m}=1$ and polynomial order $p=1$, which leads to $k, l, m=0, \ldots, 1$.

$k, l, m=0,1$ are known by [Ghanem and Spanos 1991] according to Table 2. With the resulting PC coefficients

$$
\widehat{Q}_{0}=\hat{\sigma}_{0}^{2}+\hat{\sigma}_{1}^{2}=64.25 \mathrm{GPa}^{2}, \quad \widehat{Q}_{1}=2 \hat{\sigma}_{0} \hat{\sigma}_{1}=8 \mathrm{GPa}^{2}
$$

the PCE based QoI $Q(\omega)$ in (17) becomes

$$
Q(\omega)=\widehat{Q}_{0}+\widehat{Q}_{1} \theta(\omega)=64.25 \mathrm{GPa}^{2}+8 \mathrm{GPa}^{2} \theta(\omega) .
$$

In Figure 2, right, the distribution of the random variable in (27) is illustrated as a solid line, where in addition the histogram represents the QoI $Q(\omega)$ obtained from $n_{\mathrm{MC}}=10^{8}$ Monte Carlo samples.

Finally, results for surrogate QoIs using the PCE in (20)-(22) with $n_{P}=1$ and $\mathrm{MC}$ in (7)-(9) with $n_{\mathrm{MC}}=10^{4}$ and $n_{\mathrm{MC}}=10^{8}$ are summarized in Table 3. In addition to the quantitative values of three surrogate QoIs $\mathbb{E}[Q]$, var $[Q]$, and $\mathbb{E}\left[Q^{2}\right]$, the corresponding computational times $t_{C}$ are presented. It can be seen that the PCE based results are in good agreement with MC based results and show convergence 


\begin{tabular}{|c|cccccc|}
\hline & \multicolumn{2}{|c}{$\mathrm{MC}\left(n_{\mathrm{MC}}=10^{4}\right)$} & \multicolumn{2}{c|}{$\mathrm{MC}\left(n_{\mathrm{MC}}=10^{8}\right)$} & \multicolumn{2}{c|}{$\mathrm{PCE}\left(n_{p}=1\right)$} \\
$Q^{\omega}$ & $Q^{\omega}$ & $t_{C} / \mathrm{ms}$ & $Q^{\omega}$ & $t_{C} / \mathrm{ms}$ & $Q^{\omega}$ & $t_{C} / \mathrm{ms}$ \\
\hline $\mathbb{E}[Q] / \mathrm{GPa}^{2}$ & 64.3062 & 0.375 & 64.2494 & 126.1 & 64.25 & 13.8 \\
$\operatorname{var}[Q] / \mathrm{GPa}^{4}$ & 64.3729 & 0.841 & 64.1268 & 226.3 & 64 & 23.6 \\
$\mathbb{E}\left[Q^{2}\right] / \mathrm{GPa}^{4}$ & 4199.6 & 0.542 & 4192.1 & 218.1 & 4192.7 & 21.8 \\
\hline
\end{tabular}

Table 3. Surrogate QoIs and computational times $t_{C}$ for MC with $n_{\mathrm{MC}}=10^{4}$, MC with $n_{\mathrm{MC}}=10^{8}$, and PCE with $n_{P}=1$.

after $n_{\mathrm{MC}}=10^{8}$ realizations. The increase to $n_{\mathrm{MC}}=10^{9}$, which is not shown here, results in a similar behavior.

\section{Hybrid fuzzy-stochastic analysis}

3.1. Fuzzy set and fuzzy number. So far, the design space $\mathscr{S} \subset \mathbb{R}^{n_{s}}$ in (1) has been assumed to be a precise set or a fundamental set of ordered pairs. However, in the case of a lack of knowledge also known as epistemic uncertainty, this is not realistic. Therefore, in the sequel, modeling of epistemic uncertainty is accounted for by the fuzzy analysis. To this end, the precise set $\mathscr{Y}$ in (1) is replaced by a nonprecise set, or fuzzy set [Möller and Beer 2004], respectively, of ordered pairs

$$
\begin{aligned}
& \text { 1. } \widehat{\widehat{\varphi}}=\left\{\left(\underline{s}, \mu_{S}(\underline{s})\right) \mid \underline{s} \in \mathbb{R}^{n_{s}}, \mu_{S}(\underline{s})=\min _{i=1, \ldots n_{s}}\left\{\mu \varphi_{i}\left(\underline{s}_{i}\right)\right\}\right\}, \\
& \text { 2. } \widehat{\mathscr{Y}}_{i}=\left\{\left(s_{i}, \mu \mathscr{S}_{i}\left(s_{i}\right)\right) \mid s_{i} \in \mathbb{R}, \mu_{\varphi_{i}}\left(s_{i}\right) \in[0,1]\right\}, \\
& \text { 3. } \mu \mathscr{\varphi}_{i}: \mathbb{R} \rightarrow[0,1], \quad s_{i} \mapsto \mu \mathscr{S}_{i}\left(\underline{s}_{i}\right) .
\end{aligned}
$$

According to $(28)_{1} \widehat{\mathscr{S}}$ comprises all combinations of the design variables $s_{1}, \ldots, s_{n_{s}}$ of the fuzzy sets $\widehat{\mathscr{Y}}_{i}$ in $(28)_{2}$. The corresponding function $\mu \varphi_{i}\left(s_{i}\right)$ in $(28)_{3}$ describes the degree of membership of $\underline{s}_{i} \in \mathscr{Y}_{i} \subset \mathbb{R}$ and therefore is called the membership function. In this work only normalized membership functions with the property $\sup _{s_{i} \in \mathscr{S}_{i}}\left[\mu \varphi_{i}\left(s_{i}\right)\right]=1$ are considered. A triangular fuzzy number [Möller and Beer 2004]

$$
\widehat{\mathscr{Y}}_{i}=\left\langle s_{i}^{L}, s_{i}^{M}, s_{i}^{R}\right\rangle, \quad \text { where } s_{i}^{L}<s_{i}^{M}<s_{i}^{R},
$$

is a special case of a fuzzy set and is illustrated in Figure 3, left. The stochastic QoI $Q(\underline{\kappa}(\omega, \underline{s}))$ in (6) becomes a function of the fuzzy design variables $\underline{s}$ and can be treated as a hybrid fuzzy-stochastic function. In order to determine $Q(\underline{\kappa}(\omega, \underline{s}))$, we introduce a novel hybrid fuzzy-stochastic model based on the polynomial chaos expansion. In this way, the fuzzy-random material parameter vector $\underline{\kappa}(\omega, \underline{s})$ in (4) propagates the fuzzy-random output variable $Q(\underline{\kappa}(\omega, \underline{s}))$ in (6) or the fuzzy output variable $Q^{\omega}(\underline{\kappa}(\omega, \underline{s}))$ in $(7)-(10)$. 


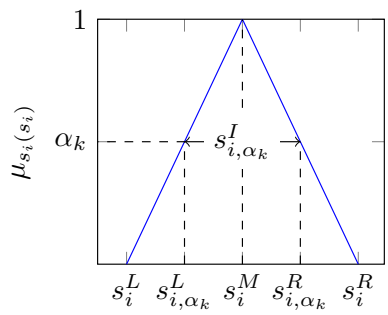

$s_{i}$

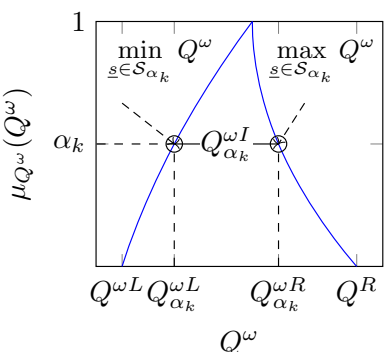

$Q^{\omega}$

Figure 3. Schematic graph of fuzzy analysis with $\alpha$-level optimization from (left) design variable (triangular) to (right) QoI.

With $\mathscr{Y}$ in (1) replaced by $\widehat{\mathscr{S}}$ in (28) the hybrid fuzzy-stochastic representation (4) for the input quantity $\underline{\kappa}(\omega, \underline{s})$ of the state and target problem in (5) and (11) accounts for the most general case in which every single component $\kappa_{i}(\omega, \underline{s})$ can be subjected to polymorphic uncertainty. Depending on the given uncertainty type of $\underline{\kappa}(\omega, \underline{s})$ in (4), the following five scenarios of uncertainty analysis (of PCE based hybrid fuzzy-stochastic analysis) can be distinguished:

- deterministic analysis

$$
\underline{\kappa}=\underline{\hat{\kappa}}=\underline{\hat{\kappa}}_{0}, \quad n_{P}=0, \quad n_{s}=0,
$$

where each material parameter $\kappa_{i}=\hat{\kappa}_{i}=\hat{\kappa}_{i 0}, i=1, \ldots, n_{m}$, is deterministic,

- stochastic analysis

$\underline{\kappa}=\underline{\kappa}(\omega), \quad \kappa_{i}=\kappa_{i}(\omega), \quad n_{P}>0, \quad n_{s}=0, \quad$ and deterministic $\underline{\hat{\kappa}}$,

where at least one material parameter is a random variable,

- fuzzy analysis

$$
\underline{\kappa}=\underline{\kappa}(\underline{s}), \quad \kappa_{i}=\kappa_{i}(\underline{s}), \quad n_{P}=0, \quad n_{s}>0,
$$

where at least one material parameter is a fuzzy variable,

- fuzzy-stochastic analysis

$$
\underline{\kappa}=\underline{\kappa}(\omega, \underline{s}), \quad \kappa_{i}=\kappa_{i}(\omega), \quad \kappa_{j}=\kappa_{j}(\underline{s}), \quad n_{P}>0, \quad n_{s}>0,
$$

where at least one material parameter is a random variable and one parameter is a fuzzy variable,

- hybrid fuzzy-stochastic analysis

$$
\underline{\kappa}=\underline{\kappa}(\omega, \underline{s}), \quad \kappa_{i}=\kappa_{i}(\omega, \underline{s}), \quad n_{P}>0, \quad n_{s}>0,
$$

where at least one material parameter is a (polymorphic uncertain) fuzzyrandom variable. 
We recall that in (30)-(34) $n_{s}$ is the number of design variables of $\underline{s}$ in (1), $n_{m}$ is the number of material parameters of $\underline{\kappa}(\omega, \underline{s})$ in (4), and $n_{P}$ is the number of PC truncations in (12).

3.2. $\alpha$-level discretization and optimization. To avoid significant numerical effort the determination of a fuzzy output can be approximated by the so-called $\alpha$-level discretization, which represents fuzzy sets numerically by $\alpha$-cuts as

$$
\mathscr{Y}_{i, \alpha_{k}}=\left\{s_{i} \mid \mu \varphi_{i, \alpha_{k}}\left(s_{i}\right) \geq \alpha_{k}, s_{i} \in \mathscr{Y}_{i}\right\}=\left[s_{i, \alpha_{k}}^{L}, s_{i, \alpha_{k}}^{R}\right]:=s_{i, \alpha_{k}}^{I}, \quad i=1, \ldots, n_{s} .
$$

The $\alpha$-cuts are characterized by lower and upper bounds $s_{i, \alpha_{k}}^{L}$ and $s_{i, \alpha_{k}}^{R}$ and therefore define crisp intervals. Figure 3, left, illustrates the crisp interval $s_{i, \alpha_{k}}^{I}$ and the membership function $\mu \mathscr{S}_{i}\left(\underline{s}_{i}\right)$ discretized by $n_{\alpha} \alpha$-levels. In this context, $0 \leq \alpha_{k} \leq 1$ denotes the membership level; see, e.g., [Möller and Beer 2004]. All fuzzy design variables $s_{i}$ are discretized using the same $\alpha$-level structure, i.e., the number and the increments of $\alpha$-levels, to form the associated crisp sets. Therefore, intervals in (35) lead to an $n_{s}$-dimensional constrained design space

$$
\mathscr{S}_{\alpha_{k}}=\left[\mathscr{S}_{1, \alpha_{k}} \times \cdots \times \mathscr{Y}_{i, \alpha_{k}} \times \cdots \times \mathscr{Y}_{n_{s}, \alpha_{k}}\right] \subset \mathscr{Y} \subset \mathbb{R}^{n_{s}} .
$$

The determination of the minimum and maximum values of the surrogate QoI in (11) or (23), respectively, at each $\alpha$-level requires the solutions of

two hybrid fuzzy-stochastic $\alpha$-level optimization problems:

1. find $Q_{\alpha_{k}}^{\omega L}=\min _{\underline{s} \in \mathscr{S}_{\alpha_{k}}} Q^{\omega}(\underline{s}, \boldsymbol{\varepsilon}(\underline{\kappa}(\omega, \underline{s}))) \approx \min _{\underline{s} \in \mathscr{S}_{\alpha_{k}}} Q^{\omega}(\underline{s}, \underline{\hat{\boldsymbol{\varepsilon}}}(\underline{s}, \underline{\hat{\kappa}}), \underline{d})$,

2. find $Q_{\alpha_{k}}^{\omega L}=\max _{\underline{s} \in \mathscr{S}_{\alpha_{k}}} Q^{\omega}(\underline{s}, \boldsymbol{\varepsilon}(\underline{\kappa}(\omega, \underline{s}))) \approx \max _{\underline{s} \in \mathscr{I}_{\alpha_{k}}} Q^{\omega}(\underline{s}, \underline{\hat{\boldsymbol{\varepsilon}}}(\underline{s}, \underline{\hat{\kappa}}), \underline{d})$.

As visualized in Figure 3, right, the two extrema $Q_{\alpha_{k}}^{\omega L}$ and $Q_{\alpha_{k}}^{\omega R}$ render two points of the membership function $\mu_{Q}(Q)$ for the membership level $\alpha_{k}$. The interval $Q_{\alpha_{k}}^{\omega I}=\left[Q_{\alpha_{k}}^{\omega L}, Q_{\alpha_{k}}^{\omega R}\right]$ is fully described by the lower and upper bounds $Q_{\alpha_{k}}^{\omega L}$ and $Q_{\alpha_{k}}^{\omega R}$. As no requirements are formulated for the continuous mapping model, both optimization problems in (37) involve only simple constraints. Problems of this kind are discussed in detail in [Bertsekas 1982].

3.3. Numerical implementation. In principle, fuzzy and stochastic dominated approaches with polymorphic uncertainties are conceivable [Reuter et al. 2012] for numerical determination of the target (11) or (23). The two approaches are illustrated in Figure 4, top and bottom. The difference between both approaches is the sequences of the evaluations of uncertainties.

For the fuzzy dominated approach, the stochastic analysis is performed inside the fuzzy analysis, which leads to $\alpha$-level optimization loops (sequence: fuzzy 


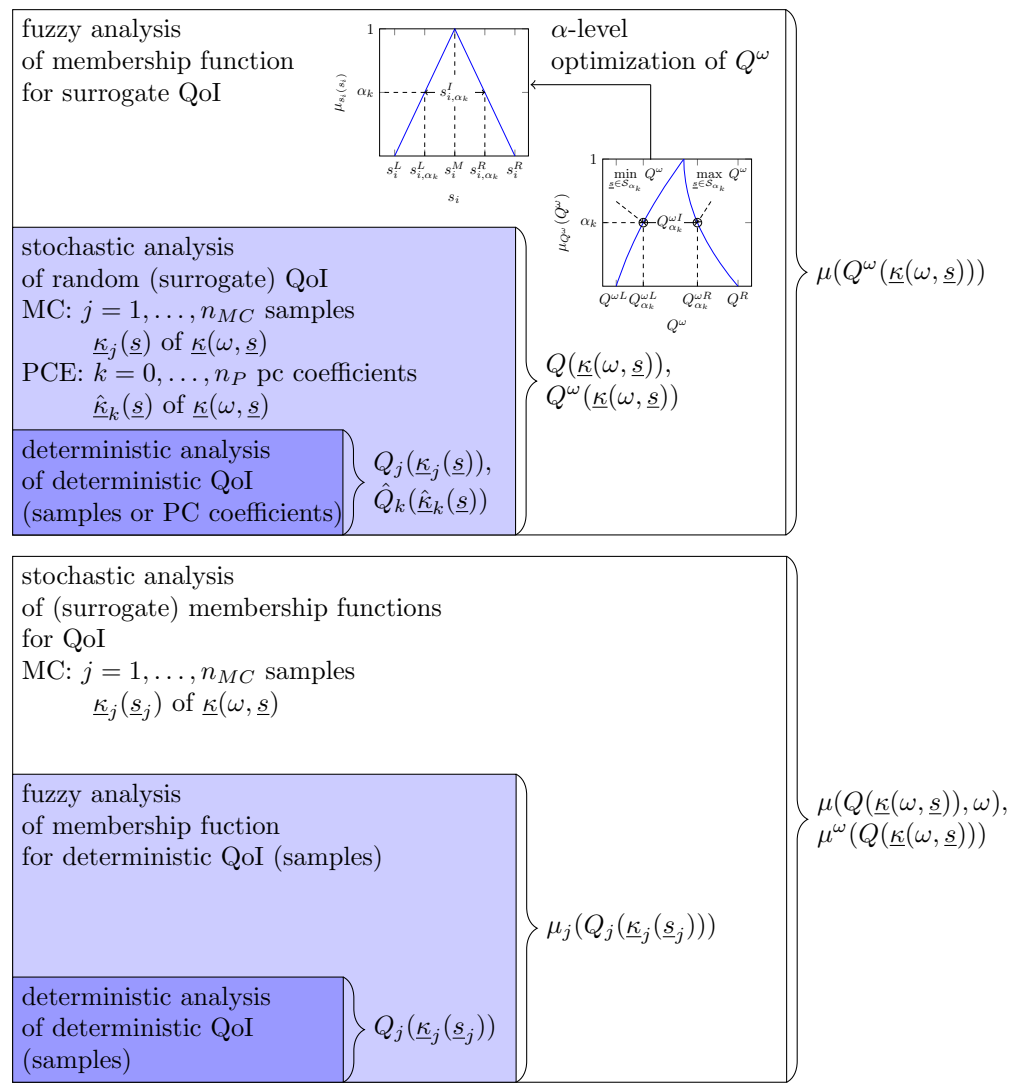

Figure 4. Computational scheme for the determination of QoIs with polymorphic uncertain parameters: (top) fuzzy dominated approach and (bottom) stochastic dominated approach.

analysis) (see (37)) with fuzzy-random variables $\underline{\kappa}(\omega, \underline{s})$. Within each iteration of the optimization loop a certain number $n_{\mathrm{MC}}$ of samples $\underline{\kappa}_{j}(\underline{s})$ (e.g., MC method) or PC coefficients $\hat{\kappa}_{k}(\underline{s})$ (PCE method) are generated (sequence: stochastic analysis). Then, samples $Q_{j}(\underline{s})$ or PC coefficients $\hat{Q}_{k}(\underline{s})$ for a QoI $Q(\underline{\kappa}(\omega, \underline{s}))$, e.g., (6), are calculated (sequence: deterministic analysis). After that, surrogate QoIs in (7)(10) or the discrete PCE based surrogate QoIs in (20)-(22) (sequence: stochastic analysis) are determined and used for the $\alpha$-level optimization (sequence: fuzzy analysis). Note, with a specific choice of a surrogate QoI, the $\alpha$-level optimization problems at each $\alpha$-level will be solved only once using the fuzzy dominated approach. The required design variables $\underline{s}$ for the solution of the membership function for (surrogate) QoIs will be used for further calculation of, e.g., stresses or strains.

In contrast, the stochastic dominated approach, where the fuzzy analysis is performed inside the stochastic analysis, leads to a certain number $n_{\mathrm{MC}}$ (sequence: 
stochastic analysis) of optimization loops (sequence: fuzzy analysis) with deterministic samples, e.g., $\underline{\kappa}_{j}$ of $\underline{\kappa}(\omega)$ (sequence: deterministic analysis). The required design variables $\underline{s}_{j}$ for the solution of the membership function for each QoI sample $\mu_{j}\left(Q_{j}\left(\underline{\kappa}_{j}, \underline{s}_{j}\right)\right)$ may be used for further calculation of interested quantity samples, e.g., samples of stresses or strains. After the fuzzy deterministic analysis, the outer stochastic analysis leads to stochastic distributions of membership functions $\mu$ or to surrogate membership functions $\mu^{\omega}$, respectively.

In this paper, the hybrid fuzzy-stochastic analysis is performed by the PCE based surrogate model, which directly provides the calculation of the surrogate QoIs in (20)-(22) and the required design variables. Therefore, and due to the small number of optimizations loops, only one fuzzy optimization of the chosen surrogate QoI, fuzzy dominated approach is preferred. In contrast, in the stochastic dominated approach, the fuzzy optimization must be performed for all samples $n_{\mathrm{MC}}$. The MC simulation, in the fuzzy dominated and also in the stochastic dominated approaches, is also performed for each example to verify the accuracy of the results, as well as the computational time.

\section{Experimental investigation of fiber reinforced plastics}

In order to describe unidirectional fiber reinforced plastics (FRP), a transversely isotropic elasticity model is used, where the plane normal to the fiber direction can be considered as an isotropic plane. In Figure 5, the fibers are aligned with the 1 -axis, which is normal to the 2-3-plane of isotropy.

In Voigt notation, the constitutive equation (3) for transversely isotropic elasticity reads

1. $\underline{\sigma}=\underline{C} \underline{\varepsilon}, \quad$ where

2. $\underline{\sigma}=\left[\sigma_{11}, \sigma_{22}, \sigma_{33}, \sigma_{12}, \sigma_{13}, \sigma_{23}\right]^{T}, \quad \underline{\varepsilon}=\left[\varepsilon_{11}, \varepsilon_{22}, \varepsilon_{33}, \gamma_{12}, \gamma_{13}, \gamma_{23}\right]^{T}$,

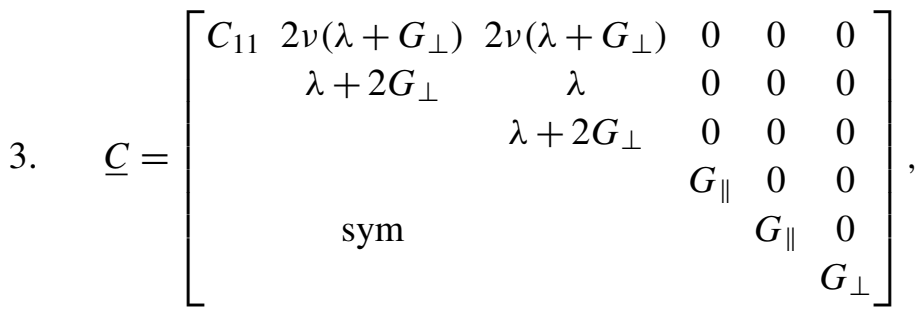

4. $\quad C_{11}=\frac{E_{\|}^{2}\left(E_{\perp}-4 G_{\perp}\right)}{4 E_{\perp} G_{\perp} v^{2}+E_{\|} E_{\perp}-4 E_{\|} G_{\perp}}$,

5. $\lambda=-\frac{2 G_{\perp}\left(2 E_{\perp} G_{\perp} v^{2}+E_{\|} E_{\perp}-2 E_{\|} G_{\perp}\right)}{4 E_{\perp} G_{\perp} v^{2}+E_{\|} E_{\perp}-4 E_{\|} G_{\perp}}$. 


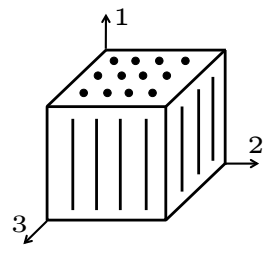

Figure 5. A transversely isotropic volume element, where fibers are aligned with the 1-axis.

In addition to the Voigt notation in $(38)_{2}$ of the stress tensor $\sigma$, the Mandel notation

$$
\underline{\sigma}_{M}=\left[\sigma_{11}, \sigma_{22}, \sigma_{33}, \sqrt{2} \sigma_{12}, \sqrt{2} \sigma_{13}, \sqrt{2} \sigma_{23}\right]^{T}
$$

is introduced, whereby (6) reads

$$
Q(\underline{\varepsilon}(\underline{\kappa}(\omega, \underline{s})))=\underline{\sigma}_{M}^{T}(\underline{\varepsilon}(\underline{\kappa}(\omega, \underline{s}))) \underline{\sigma}_{M}(\underline{\varepsilon}(\underline{\kappa}(\omega, \underline{s}))) .
$$

In (38) $v$ is the dimensionless Poisson ratio and $E_{\|}, E_{\perp}$ and $G_{\|}, G_{\perp}$ are Young's moduli and shear moduli in fiber and transverse direction, respectively. According to (4), the $n_{m}=5$ required transversely isotropic material parameters are summarized in the material parameter vector

$$
\underline{\kappa}=\left[\kappa_{1}, \kappa_{2}, \kappa_{3}, \kappa_{4}, \kappa_{5}\right]^{T}=\left[E_{\|}, E_{\perp}, G_{\|}, G_{\perp}, \nu\right]^{T} .
$$

The determination of the shear modulus $G_{\perp}$ in the isotropic plane requires a shear test apparatus, which currently is not available. Instead only tensile tests can be performed, which are used to determine the material parameters in (41), except $G_{\perp}$, experimentally.

To this end, three plates made of unidirectional FRP with different fiber orientations $\left(0^{\circ}, 45^{\circ}, 90^{\circ}\right)$ were produced. From each plate 30 tensile specimens were cut out. The geometry of the tensile specimen is depicted in Figure 6b. The width $b_{0}$ and the thickness $t_{0}$ of the specimens differ from $0^{\circ}$ orientation to $45^{\circ}$ and $90^{\circ}$ orientation, where the dimensions for the different fiber orientations are summarized in Table 4. Furthermore, Figure 6c-e shows schematically the different fiber orientations of the specimens. These are clamped into a tensile testing machine with hydraulic clamping jaws as illustrated in the experimental setup in Figure 6a. The FRP is subjected to an off-axis uniaxial stress loading, in which the fibers are oriented in the 1-direction, and rotated around the 3-direction by an angle $\varphi$. With respect to the testing machine a machine-fixed coordinate system $(x, y)$ in Figure 6 is defined. The experiments, which are displacement controlled according to DIN EN ISO 527-5, are loaded in the longitudinal direction $x$ at an angle $\varphi$ with respect to the fiber direction. In particular, $\varphi=0^{\circ}$ and $\varphi=90^{\circ}$ correspond to longitudinal and transverse uniaxial stress loading, respectively. A load cell supplies forces $\bar{F}$ 
(a)

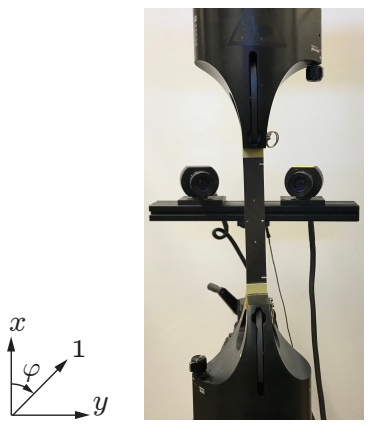

(b)

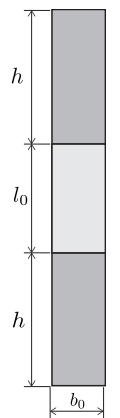

(c)

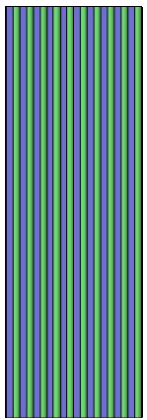

(d)

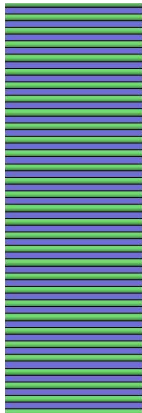

(e)

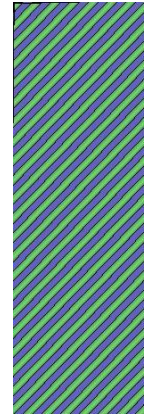

Figure 6. Experimental investigation: (a) experimental setup, (b) geometry of specimens, and schematic specimens for fiber orientation (c) $0^{\circ}$, (d) $90^{\circ}$, and (e) $45^{\circ}$. Machine-fixed coordinate system $(x, y)$ and fibers aligned to 1 -axis.

\begin{tabular}{|l|rr|}
\hline$[\mathrm{mm}]$ & $0^{\circ}$ & $45^{\circ} / 90^{\circ}$ \\
\hline length $l_{0}$ & 150 & 150 \\
clamping length $h$ & 50 & 50 \\
width $b_{0}$ & 15 & 25 \\
thickness $t_{0}$ & 1 & 2 \\
\hline
\end{tabular}

Table 4. Measures of specimens depending on fiber orientations.

at different observation states, whereby corresponding stresses in longitudinal direction $\bar{\sigma}_{x}=\bar{F} / A_{0}$ are determined using the cross-sectional area $A_{0}=b_{0} t_{0}$; see Table 4. By applying a video extensometer, the strains in longitudinal and transverse directions $\bar{\varepsilon}_{x}$ and $\bar{\varepsilon}_{y}$ are measured.

Fiber orientation $\varphi=0^{\circ}$. In order to determine the Young's modulus $E_{\|}$in fiber direction as well as the Poisson's ratio $v$, tensile tests are applied for samples illustrated in Figure 6c. DIN EN ISO 527-1 provides further information regarding the test procedure and the data evaluation. The resulting curves for stress $\sigma_{11}=\bar{\sigma}_{x}$ versus strain $\varepsilon_{11}=\bar{\varepsilon}_{x}$ for 30 experiments are shown in Figure 7, left, where the slope of each curve describes the corresponding Young's modulus $E_{\|}=\Delta \sigma_{11} / \Delta \varepsilon_{11}$ in fiber direction. Furthermore, in Figure 7, right, experimental longitudinal strain $\varepsilon_{11}$ versus transversal strain $\left|\varepsilon_{22}\right|=\left|\bar{\varepsilon}_{y}\right|$ curves are illustrated, where the slope of each regression line for each curve renders the corresponding Poisson's ratio $v=$ $-\Delta \varepsilon_{22} / \Delta \varepsilon_{11}$. The frequency distributions of $E_{\|}$and $v$ are illustrated in Figure 8, left and right. 

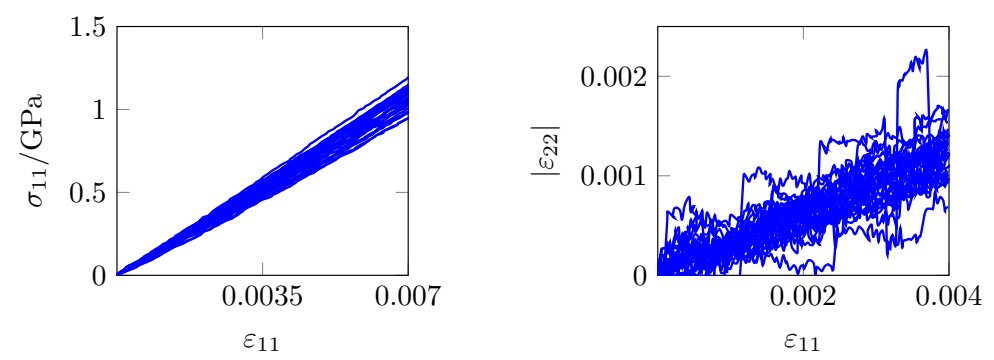

Figure 7. Experimental results for fiber orientation $0^{\circ}$ : (left) longitudinal stress $\sigma_{11}$ versus longitudinal strain $\varepsilon_{11}$ curves and (right) longitudinal strain $\varepsilon_{11}$ versus transversal absolute strain $\left|\varepsilon_{22}\right|$ curves.
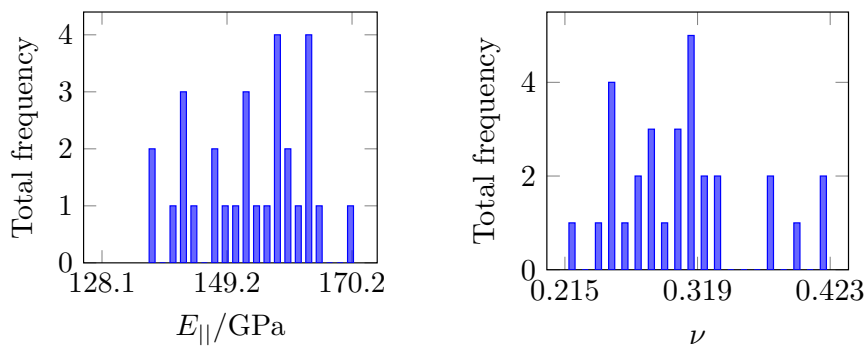

Figure 8. Frequency distributions of identified parameters for fiber orientation $0^{\circ}$ : (left) Young's modulus in fiber direction $E_{\|}$ and (right) Poisson's ratio $v$.

Fiber orientation $\varphi=90^{\circ}$. To determine the Young's modulus $E_{\perp}$ transverse to the fiber direction, tensile tests are applied for samples illustrated in Figure 6d. DIN EN ISO 527-1 provides further information regarding the test procedure and the data evaluation. Results for 30 experimental stress $\sigma_{22}=\bar{\sigma}_{x}$ versus strain $\varepsilon_{22}=\bar{\varepsilon}_{x}$ curves are illustrated in Figure 9, left, where the slope of each curve renders the corresponding Young's modulus $E_{\perp}=\Delta \sigma_{22} / \Delta \varepsilon_{22}$. The frequency distribution of $E_{\perp}$ is illustrated in Figure 9, right.

Fiber orientation $\varphi=45^{\circ}$. In order to determine the shear modulus $G_{\|}$in fiber direction, tensile tests according to DIN EN ISO 527-5 are applied for samples illustrated in Figure 6e. Results for 30 experimental maximal shear stress $\sigma_{12}=\bar{\sigma}_{x} / 2$ versus shear strain $\gamma_{12} \approx \bar{\varepsilon}_{x}-\bar{\varepsilon}_{y}$, according to DIN EN ISO 14129, are illustrated in Figure 10, left, where the slope of each regression line for each curve renders the corresponding shear modulus $G_{\|}=\Delta \sigma_{12} / \Delta \gamma_{12}$. The frequency distribution of $\mathrm{j} G_{\|}$is illustrated in Figure 10, right. 

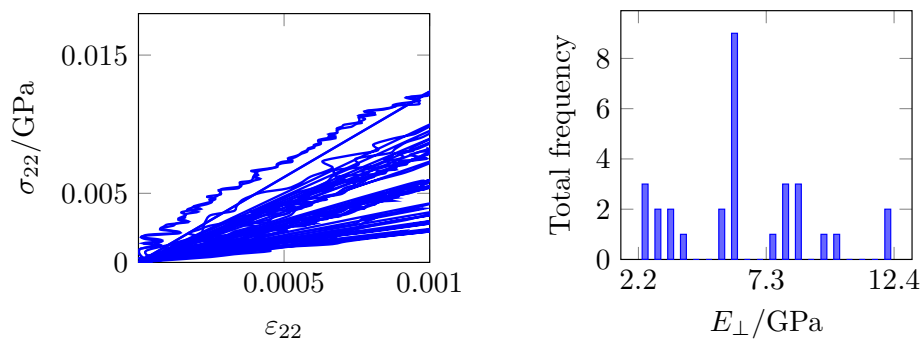

Figure 9. Experimental results and identification for fiber orientation $90^{\circ}$ : (left) curves for stress $\sigma_{22}$ transversal to fiber versus strain $\varepsilon_{22}$ transversal to fiber and (right) frequency distribution of Young's modulus in transverse direction $E_{\perp}$.
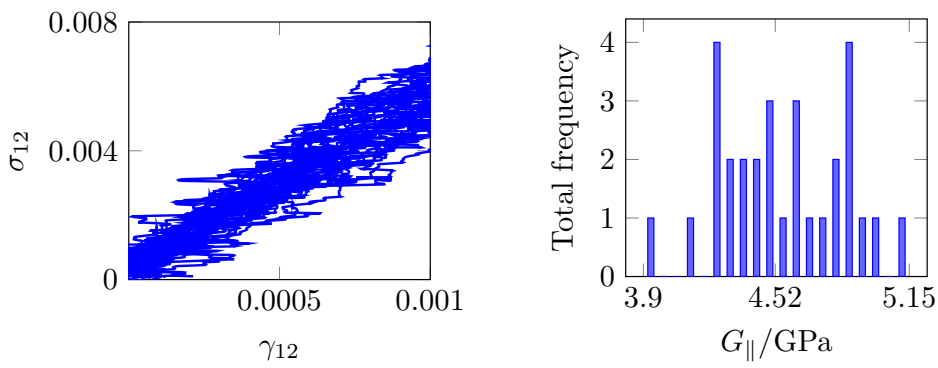

Figure 10. Experimental results and identification for fiber orientation $45^{\circ}$ : (left) maximal shear stress $\sigma_{12}$ versus maximal shear strain $\gamma_{12}$ curves and (right) frequency distribution of shear modulus in fiber direction $G_{\|}$.

\section{Representative numerical examples}

For the following strain controlled examples, fuzzy analysis, fuzzy-stochastic analysis, and hybrid fuzzy-stochastic analysis, the strain vector is given as

$$
\underline{\varepsilon}=\left[\varepsilon_{11}, \varepsilon_{22}, \varepsilon_{33}, \gamma_{12}, \gamma_{13}, \gamma_{23}\right]^{T}=[0.05,0.02,0.03,0.005,0.004,0.002]^{T} .
$$

As mentioned in Section 4, we are currently not able to determine the shear modulus in the isotropic plane. Therefore, $G_{\perp}=3.9286 \mathrm{GPa}$ is taken from the literature according to [Soden et al. 1998] and is regarded as deterministic.

5.1. Fuzzy analysis. In the first representative example, all material parameters except $G_{\perp}$ in (41) are assumed as purely epistemic uncertain. Since $G_{\perp}$ is deterministic it can be considered as a degenerated fuzzy variable in Figure 11e. The (fuzzy) input according to (32) includes no (stochastic) random variables and is 
(a)

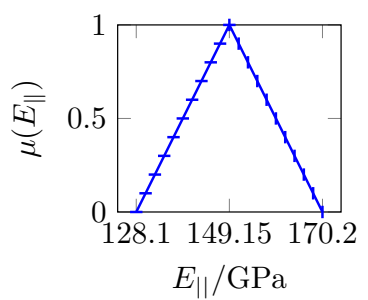

(d)

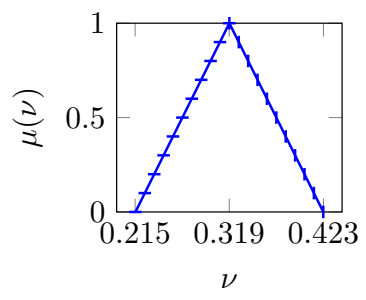

(b)

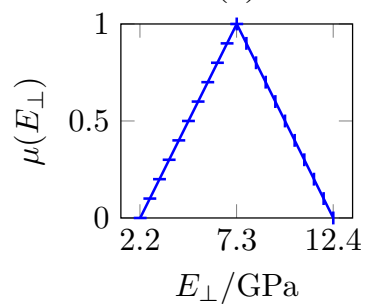

(c)

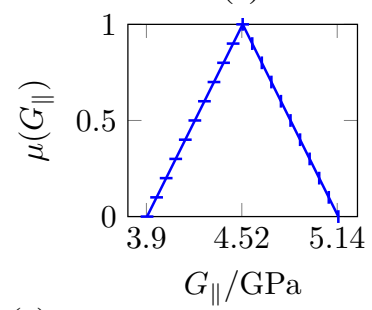

(e)

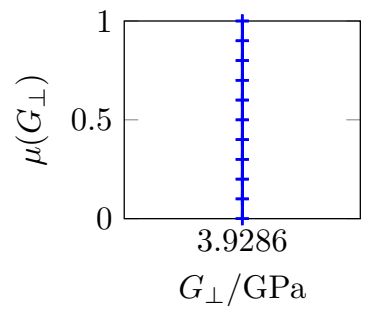

Figure 11. Fuzzy analysis: input membership functions for material design variables (a) $E_{\|}$, (b) $E_{\perp}$, (c) $G_{\|}$, (d) $v$, and (e) $G_{\perp}$.

defined as

$$
\underline{\kappa}(\underline{s})=\left[E_{\|}(\underline{s}), E_{\perp}(\underline{s}), G_{\|}(\underline{s}), G_{\perp}, v(\underline{s})\right]^{T}=\left[s_{1}, s_{2}, s_{3}, G_{\perp}, s_{4}\right]^{T},
$$

such that the vector in (1) of $n_{s}=4$ design variables for $n_{m}=5$ material parameters is given by

$$
\underline{s}=\left[s_{1}, s_{2}, s_{3}, s_{4}\right]^{T}=\left[E_{\|}, E_{\perp}, G_{\|}, \nu\right]^{T} .
$$

In the special case of fuzzy analysis according to (32), the matrix of PC coefficients in (16) reduces with $n_{P}=0$ to a vector corresponding to the material parameter vector in (43)

$$
\underline{\hat{\kappa}}(\underline{s})=\underline{\hat{\kappa}}_{0}(\underline{s})=\underline{\kappa}(\underline{s}) .
$$

As discussed in [Möller and Beer 2004] several possible membership functions can be laid over the frequency distributions. This may depend, e.g., on the available data. Since there are no experimental results available for validation in the current work, we decided to use simple triangular shape functions by normalizing the memberships. Based on the identified parameters represented as frequency distributions in Figures 8; 9, right; and 10, right; membership functions $\mu(\underline{s})$ are generated for the input quantities $E_{\|}, E_{\perp}, G_{\|}$, and $v$ according to Figure 11a-d. To this end, the empirical mean $\mathbb{E}\left[s_{i}^{*}\right]$ of the identified parameter samples $s_{i}^{*}$ are chosen as mean values $s_{i}^{M}$ to obtain triangular fuzzy numbers according to (29) as

$$
\widehat{\mathscr{Y}}_{i}=\left\langle s_{i}^{L}, s_{i}^{M}, s_{i}^{R}\right\rangle=\left\langle\mathbb{E}\left[s_{i}^{*}\right]-f_{i}^{*}, \mathbb{E}\left[s_{i}^{*}\right], \mathbb{E}\left[s_{i}^{*}\right]+f_{i}^{*}\right\rangle,
$$


where $f_{i}^{*}=\max \left\{\mathbb{E}\left[s_{i}^{*}\right]-\min s_{i}^{*}, \max s_{i}^{*}-\mathbb{E}\left[s_{i}^{*}\right]\right\}$, that is, for each material parameter a maximum deviation of $f_{i}^{*}$ for the epistemic uncertainty is assumed. Thus, the triangular fuzzy numbers

$$
\begin{array}{lll}
\text { 1. } & \widehat{\mathscr{S}}_{1}=\langle 128.1,149.15,170.2\rangle \mathrm{GPa}, & f_{1}^{*}=21.05 \mathrm{GPa}, \\
\text { 2. } & \widehat{\mathscr{S}}_{2}=\langle 2.2,7.3,12.4\rangle \mathrm{GPa}, & f_{2}^{*}=5.1 \mathrm{GPa}, \\
\text { 3. } & \widehat{\mathscr{S}}_{3}=\langle 3.9,4.52,5.14\rangle \mathrm{GPa}, & f_{3}^{*}=0.62 \mathrm{GPa}, \\
\text { 4. } & \widehat{\mathscr{S}}_{4}=\langle 0.215,0.319,0.423\rangle, & f_{4}^{*}=0.104
\end{array}
$$

are chosen for the four design variables in (44). The $\alpha$-discretization is performed with $n_{\alpha}=11$ cuts. These discrete input parameters define the stresses in (38) and the QoI in (40). Note that the QoI $Q(\underline{s})$ in (40) in the purely (epistemic) fuzzy analysis is independent on $\omega$ such that no surrogate QoIs are needed. The minimization and maximization problems in (37) $)_{1}$ and (37) 2 are solved for $Q(\underline{s})$ in (40) at each $\alpha$-level using a standard optimization tool for constrained functions. In this work the function fmincon with the sequential quadratic programming [Nocedal and Wright 1999] algorithm of the Matlab Optimization Toolbox is applied.

In Figure 12, the resulting output membership function of the optimization problems in (37) for the QoI $Q(\underline{s})$ presented in (40) is illustrated. The marks - and I show that from each pair of interval bounds of the design variables $s_{i, \alpha_{k}}^{L}$ and $s_{i, \alpha_{k}}^{R}$ at each $\alpha$-level $\alpha_{k}, k=1, \ldots, 11$, in Figure 11a,d, the interval bounds of the QoIs $Q_{\alpha_{k}}^{\omega L}$ and $Q_{\alpha_{k}}^{\omega R}$ are obtained. Therefore, calculating the stresses $\underline{\sigma}(\omega, \underline{s})$ in (38), the left and right interval bounds $s_{i, \alpha_{k}}^{L}$ and $s_{i, \alpha_{k}}^{R}$ at each $\alpha$-level $\alpha_{k}, k=1, \ldots, 11$, can be used.

The uncertain stress coefficients $\sigma_{i j}$ of the stress vector $\underline{\sigma}$ in (38) are illustrated in Figure 13. Due to Hooke's law described in (38) the normal stress coefficients $\sigma_{i i}(\underline{s})$ are effected by the design variables $\underline{s}=\left[E_{\|}, E_{\perp}, G_{\|}, v\right]^{T}$ and the fixed material parameter $G_{\perp}$. Remarkably, the membership functions for the normal stress coefficients $\sigma_{i i}(\underline{s})$ in Figure 13a-c become nonlinear. This is due to the nonlinear mathematical operations of fuzzy variables for the calculation of $C_{11}$ and $\lambda$ in (38).

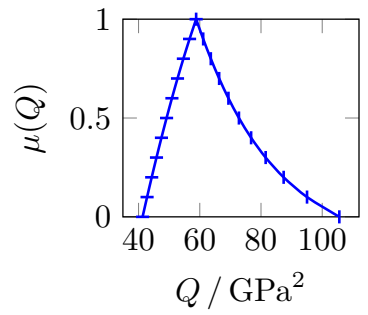

Figure 12. Fuzzy analysis: output membership function for quantity of interest $Q(\underline{\varepsilon}(\underline{s}))$ in (40). 
(a)

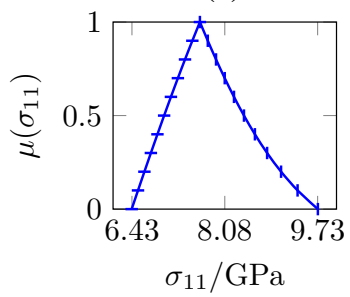

(d)

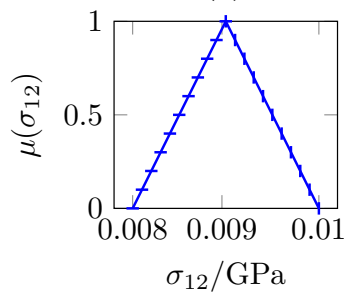

(b)

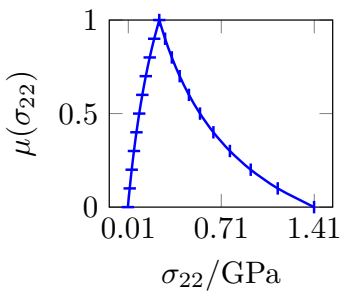

(e)

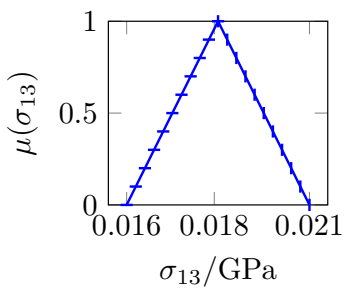

(c)

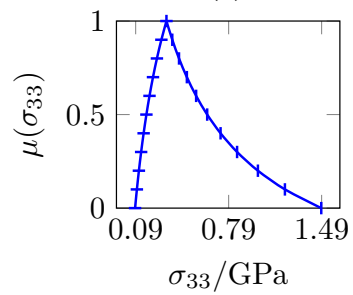

(f)

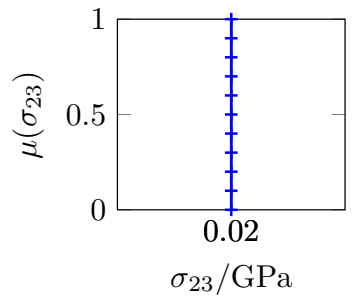

Figure 13. Fuzzy analysis: output membership functions for stresses (a) $\sigma_{11}$, (b) $\sigma_{22}$, (c) $\sigma_{33}$, (d) $\sigma_{12}$, (e) $\sigma_{13}$, and (f) $\sigma_{23}$.

The stress components $\sigma_{12}(\underline{s})$ and $\sigma_{13}(\underline{s})$ are only effected by the design variables $G_{\|}(\underline{s})$. Therefore, the membership functions $\mu\left(\sigma_{12}\right)$ and $\mu\left(\sigma_{13}\right)$ in Figure $13 \mathrm{~d}-\mathrm{e}$ have the same triangular shape as the membership function $\mu\left(G_{\|}\right)$of the design variable $G_{\|}$in Figure 11c. The shear stress $\sigma_{23}$ depends on the deterministic parameter $G_{\perp}$. Therefore, The membership function $\mu\left(\sigma_{23}\right)$ in Figure $13 \mathrm{f}$ becomes deterministic and is represented by a straight vertical line.

5.2. Fuzzy-stochastic analysis. From an industrial point of view, the mechanical properties in the fiber direction are of great interest. Therefore, in practice more experimental investigations are carried out in the fiber direction than in the transverse direction. Consequently, in the second representative example, two material parameters $E_{\|}(\omega)$ and $v(\omega)$ of the input vector in (41) are assumed as purely aleatoric, whereas $E_{\perp}(\underline{s}), G_{\|}(\underline{s})$, and $G_{\perp}=3.9286 \mathrm{GPa}$ remain epistemic and deterministic as in Section 5.1. For a fuzzy-stochastic analysis the input material parameter vector (41) according to (33) is defined as

$$
\underline{\kappa}(\omega, \underline{s})=\left[E_{\|}(\omega), E_{\perp}(\underline{s}), G_{\|}(\underline{s}), G_{\perp}, v(\omega)\right]^{T},
$$

such that the design variable vector (1) of material parameters is given by

$$
\underline{s}=\left[s_{1}, s_{2}\right]^{T}=\left[E_{\perp}, G_{\|}\right]^{T} .
$$

The stochastic parameters $E_{\|}(\omega)$ and $v(\omega)$ are assumed to be normally distributed random variables, which requires a truncation of $n_{P}=2$ in (12). The membership 
(a)

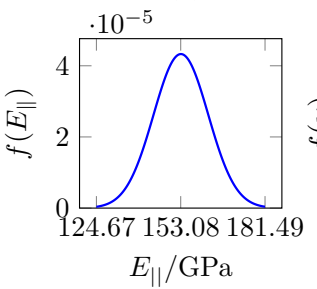

(b)

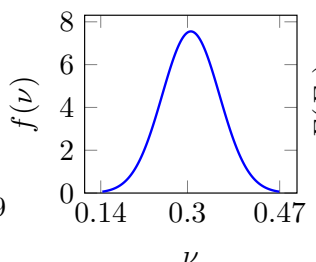

(c)

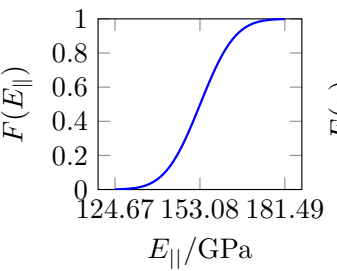

(d)

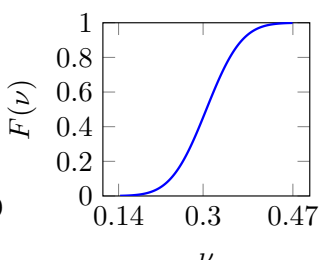

Figure 14. Fuzzy-stochastic analysis: input density functions for the aleatory material parameters (a) PDF for $E_{\|}$, (b) PDF for $v$, (c) $\mathrm{CDF}$ for $E_{\|}$, and (d) CDF for $v$.

functions $\mu(\underline{s})$ for the material parameters $E_{\perp}$ and $G_{\|}$remain unchanged according to Figure 11a,d and used as input quantities for the fuzzy analysis. Based on the frequency distributions in Figure 8 normally distributed density functions are generated from the expectation values $\mathbb{E}\left[E_{\|}(\omega)\right], \mathbb{E}[v(\omega)]$ and standard deviations $\operatorname{std}\left[E_{\|}(\omega)\right], \operatorname{std}[v(\omega)]$ for material parameters $E_{\|}(\omega), v(\omega)$, which are used as input quantities for the stochastic sequence in the fuzzy-stochastic analysis. In Figure 14 the corresponding probability density functions (PDFs) and cumulative density functions (CDFs) are given. According to (13), the PCEs for the normally distributed random variables $E_{\|}(\omega)$ and $\nu(\omega)$ for $n_{P}=2$ are

$$
\begin{aligned}
E_{\|}(\omega) & =\widehat{E}_{\| 0}+\widehat{E}_{\| 1} \theta_{1}(\omega)+\widehat{E}_{\| 2} \theta_{2}(\omega), \\
v(\omega) & =\hat{v}_{0}+\hat{v}_{1} \theta_{1}(\omega)+\hat{v}_{2} \theta_{2}(\omega) .
\end{aligned}
$$

We assume, further, that $E_{\|}(\omega)$ and $v(\omega)$ are independent random variables such that $(50)$ renders

$$
\begin{gathered}
E_{\|}(\omega)=\widehat{E}_{\| 0}+\widehat{E}_{\| 1} \theta_{1}(\omega), \\
v(\omega)=\hat{v}_{0}+\hat{v}_{2} \theta_{2}(\omega),
\end{gathered}
$$

with PC coefficients

$$
\begin{aligned}
\widehat{E}_{\| 0} & =\mathbb{E}\left[E_{\|}\right]=153.145, & \widehat{E}_{\| 1} & =\operatorname{std}\left[E_{\|}\right]=9.214, \\
\hat{v}_{0} & =\mathbb{E}[v]=0.306, & \hat{v}_{2} & =\operatorname{std}[v]=0.0528 .
\end{aligned}
$$

Consequently, the corresponding input matrix in (16) of material parameter PC coefficients becomes

$$
\hat{\kappa}(\underline{s})=\left[\begin{array}{ccc}
\widehat{E}_{\| 0} & \widehat{E}_{\| 1} & 0 \\
E_{\perp}(\underline{s}) & 0 & 0 \\
G_{\|}(\underline{s}) & 0 & 0 \\
G_{\perp} & 0 & 0 \\
\hat{v}_{0} & 0 & \hat{v}_{2}
\end{array}\right]=\left[\begin{array}{ccc}
153.145 & 9.214 & 0 \\
s_{1} & 0 & 0 \\
s_{2} & 0 & 0 \\
3.9286 & 0 & 0 \\
0.306 & 0 & 0.0528
\end{array}\right] .
$$



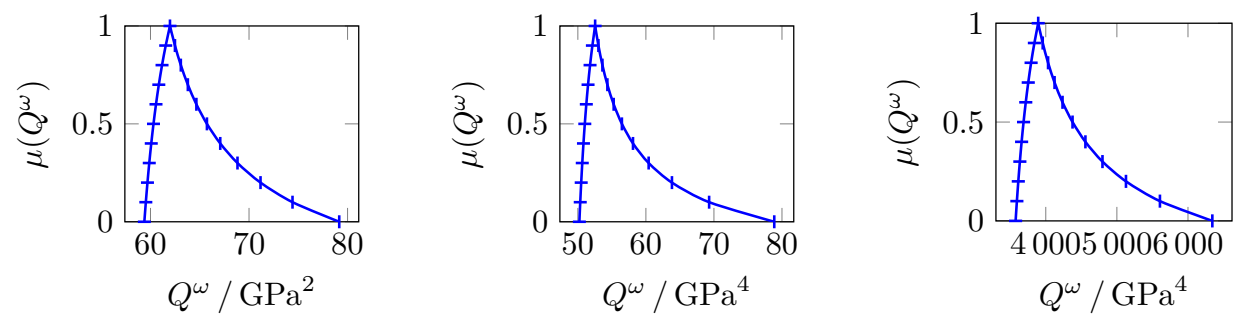

Figure 15. Fuzzy-stochastic analysis: membership function for surrogate QoIs in (20)-(22) according to QoI in (40), (left) $Q^{\omega}=$ $\mathbb{E}[Q]$, (center) $Q^{\omega}=\operatorname{var}[Q]$, and (right) $Q^{\omega}=\mathbb{E}\left[Q^{2}\right]$.

As mentioned in Section 3.3, both analyses, the fuzzy and the stochastic ones, are combined with the fuzzy dominated or stochastic dominated approach as illustrated in the computational scheme in Figure 4. Here, the focus is on the PCE based fuzzy dominated approach, in which the PC coefficients in (53), although only the 0-th, are represented as fuzzy design variables. Since the QoI in (40) will be a fuzzyrandom variable, it is necessary to choose a surrogate QoI to perform the outer fuzzy optimization introduced in Figure 4, left (sequence: fuzzy analysis).

In Figure 15, the resulting output membership functions of the optimization problems in (37) for three possible realizations of surrogate QoIs presented in (20)-(22) are illustrated. The marks - and | show that from each pair of interval bounds of the design variables $s_{i, \alpha_{k}}^{L}$ and $s_{i, \alpha_{k}}^{R}$ at each $\alpha$-level $\alpha_{k}, k=1, \ldots, 11$, in Figure $11 \mathrm{~b}-\mathrm{c}$, the interval bounds of the surrogate QoIs $Q_{\alpha_{k}}^{\omega L}$ and $Q_{\alpha_{k}}^{\omega R}$ are obtained. Therefore, for postprocessing, e.g., calculating the stresses $\underline{\sigma}(\omega, \underline{s})$ in $(38)$, the left and right interval bounds $s_{i, \alpha_{k}}^{L}$ and $s_{i, \alpha_{k}}^{R}$ at each $\alpha$-level $\alpha_{k}, k=1, \ldots, 11$, can be used.

In the fuzzy dominated approach as presented in Figure 4, left, for verification, the MC method with $n_{\mathrm{MC}}=10^{4}$ Monte Carlo samples renders similar values. Results for surrogate QoIs using the PCE in (20)-(22) with $n_{P}=2$ and MC in (7)-(9) with $n_{\mathrm{MC}}=10^{3}$ and $n_{\mathrm{MC}}=10^{4}$ are summarized in Table 5. In addition to the quantitative values of the three surrogate QoIs $\mathbb{E}[Q]$, var $[Q]$, and $\mathbb{E}\left[Q^{2}\right]$, the computational times $t_{C}$ are presented. It can be seen that in the MC solution, even with a small number of samples, the computational effort is considerably greater than with the PCE solution, although the deviations are not negligible.

Due to the Hooke's law (38) the stress components $\sigma_{12}(\underline{s})$ and $\sigma_{13}(\underline{s})$ are only effected by the design variables $G_{\|}(\underline{s})$, where $\sigma_{23}$ depends on the deterministic parameter $G_{\perp}$. Consequently, $\sigma_{12}, \sigma_{13}$, and $\sigma_{23}$ remain unchanged compared to the results in Figure $13 \mathrm{~d}-\mathrm{f}$ of the fuzzy analysis in Section 5.1. However, by (38) the normal stresses $\sigma_{i i}(\omega, \underline{s}), i=1,2,3$, are effected by design variables $E_{\perp}(\underline{s}), G_{\|}(\underline{s})$ and stochastic variables $E_{\|}(\omega), v(\omega)$. Therefore, $\sigma_{i i}(\omega, \underline{s})$ become 


\begin{tabular}{|cl|cccccccccc|}
\hline & & \multicolumn{3}{|c}{$\mathrm{MC}\left(n_{\mathrm{MC}}=10^{3}\right)$} & \multicolumn{3}{c|}{$\mathrm{MC}\left(n_{\mathrm{MC}}=10^{4}\right)$} & \multicolumn{4}{c|}{$\mathrm{PCE}\left(n_{p}=2\right)$} \\
$Q^{\omega}$ & $\alpha_{k}$ & $Q_{\alpha_{k}}^{\omega L}$ & $Q_{\alpha_{k}}^{\omega R}$ & $t_{C} / \mathrm{s}$ & $Q_{\alpha_{k}}^{\omega L}$ & $Q_{\alpha_{k}}^{\omega R}$ & $t_{C} / \mathrm{s}$ & $Q_{\alpha_{k}}^{\omega L}$ & $Q_{\alpha_{k}}^{\omega R}$ & ${ }{ }_{C} / \mathrm{s}$ \\
\hline \multirow{2}{*}{$\frac{\mathbb{E}[Q]}{\mathrm{GPa}^{2}}$} & 1 & 62.10 & 62.10 & & 61.31 & 61.31 & & 61.98 & 61.98 & \\
& 0.5 & 60.57 & 65.36 & 192 & 60.17 & 64.97 & 1916 & 60.31 & 65.73 & 3.7 \\
& 59.69 & 76.16 & & 59.29 & 75.81 & & 59.38 & 79.16 & \\
$\frac{\operatorname{var}[Q]}{\mathrm{GPa}^{4}}$ & 1 & 58.37 & 58.37 & & 53.38 & 53.38 & & 52.54 & 52.54 & \\
& 0.5 & 56.23 & 63.46 & 218 & 51.85 & 51.85 & 2011 & 51.02 & 56.47 & 3.8 \\
& 55.07 & 86.95 & & 51.03 & 76.71 & & 50.22 & 78.87 & \\
$\frac{\mathbb{E}\left[Q^{2}\right]}{\mathrm{GPa}^{4}}$ & 1 & 3856 & 3856 & & 3864 & 3864 & & 3894 & 3894 & \\
& 0.5 & 3667 & 4277 & 110 & 3675 & 4282 & 1071 & 3688 & 4377 & 3.5 \\
\hline
\end{tabular}

Table 5. Surrogate QoIs and computational times $t_{C}$ using MC with $n_{\mathrm{MC}}=10^{3}$, MC with $n_{\mathrm{MC}}=10^{4}$ and PCE with $n_{P}=2$.
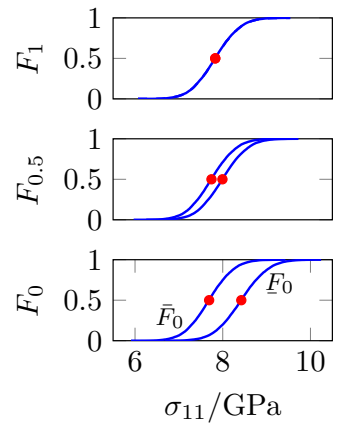
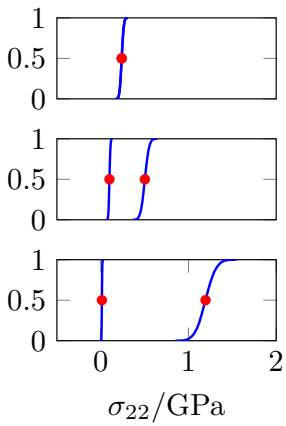
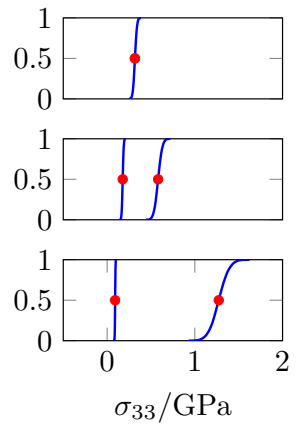

Figure 16. Fuzzy-stochastic analysis: P-boxes at $\alpha$-levels $\alpha_{1}=0$, $\alpha_{6}=0.5$ and $\alpha_{11}=1$ for normal stresses (left) $\sigma_{11}$, (center) $\sigma_{22}$, and (right) $\sigma_{33}$.

hybrid fuzzy-stochastic variables, which can be seen in Figure 16, i.e, the CDFs of $\sigma_{i i}(\omega, \underline{s})$ are not anymore given by a unique $\mathrm{CDF}$ as for a stochastic variable, e.g., in Figure $14 \mathrm{c}-$ d. Instead, they are given by left upper bounds $\bar{F}_{\alpha_{k}}\left(\sigma_{i i}\right)$ and right lower bounds $\underline{F}_{\alpha_{k}}\left(\sigma_{i i}\right)$ at each $\alpha$-level, where the intervals of CDFs $\left[\bar{F}_{\alpha_{k}}\left(\sigma_{i i}\right), \underline{F}_{\alpha_{k}}\left(\sigma_{i i}\right)\right]$ are called probability boxes (P-boxes). For further explanation on P-boxes the reader is referred to [Ferson et al. 2003]. The P-boxes of the normal stresses $\sigma_{i i}$ at three $\alpha$-levels $0,0.5$, and 1 are shown in Figure 16.

In addition, different statistical moments of the fuzzy-stochastic variables $\sigma_{i i}$ can be calculated. The expectation values $\mathbb{E}\left[\sigma_{i i}\right]$ and variances $\operatorname{var}\left[\sigma_{i i}\right]$ are illustrated in Figures 17 and 18. While, as shown in Figure 16, $\sigma_{i i}$ are fuzzy-stochastic random variables, their moments in Figures 17 and 18 are fuzzy variables. The red dots in 

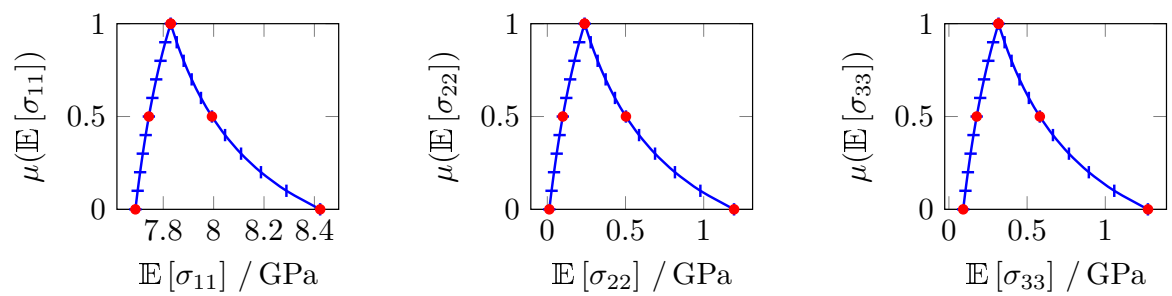

Figure 17. Fuzzy-stochastic analysis: output membership functions for expectation values of normal stresses (left) $\mathbb{E}\left[\sigma_{11}\right]$, (center) $\mathbb{E}\left[\sigma_{22}\right]$, and (right) $\mathbb{E}\left[\sigma_{33}\right]$.
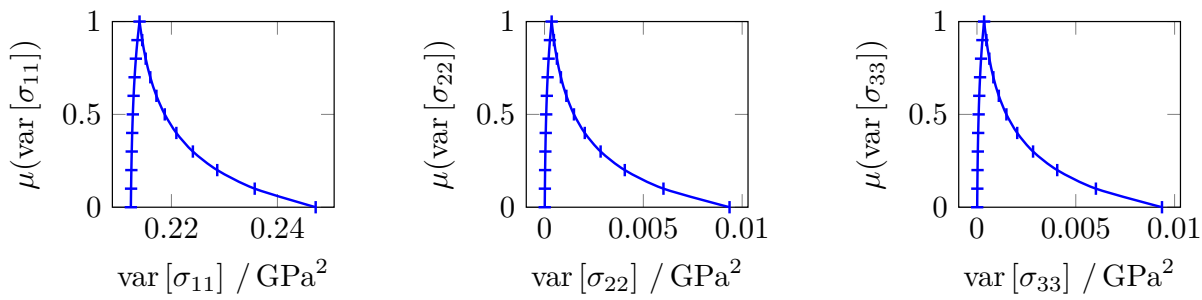

Figure 18. Fuzzy-stochastic analysis: output membership functions for variances of normal stresses (left) $\operatorname{var}\left[\sigma_{11}\right]$, (center) $\operatorname{var}\left[\sigma_{22}\right]$, and (right) $\operatorname{var}\left[\sigma_{33}\right]$.

Figure 16 indicate the expectation values of the left upper bounds $\bar{F}_{\alpha_{k}}\left(\sigma_{i i}\right)$ and right lower bounds $\underline{F}_{\alpha_{k}}\left(\sigma_{i i}\right)$ at three $\alpha$-levels $\alpha_{1}=0, \alpha_{6}=0.5$, and $\alpha_{11}=1$. The same values are also shown by red dots in Figure 17. Among others, these figures are suitable for investigating the influence of design variables $\underline{s}$ on statistical moments $\mathbb{E}\left[\sigma_{i i}\right]$ and $\operatorname{var}\left[\sigma_{i i}\right]$, respectively. Both in Figures 17 and 18 the sensitivity of the design variables $\underline{s}$ can be clearly seen. Higher values of $\underline{s}$ result in a larger increase in statistical moments than for smaller values of $\underline{s}$. The study of such influences are of great importance in industrial applications and can be used in the risk and reliability analysis.

5.3. Hybrid fuzzy-stochastic analysis. In the third representative example, all material parameters are the same as in the previous example in Section 5.2 except $E_{\|}$, which is assumed as a polymorphic uncertain material parameter $E_{\|}(\omega, \underline{s})$ as illustrated in Figure 19. For a hybrid fuzzy-stochastic analysis the input material parameter vector equation (41) is given as

$$
\underline{\kappa}(\omega, \underline{s})=\left[E_{\|}(\omega, \underline{s}), E_{\perp}(\underline{s}), G_{\|}(\underline{s}), G_{\perp}, v(\omega)\right]^{T},
$$

whereas the design variable vector equation (1) of material parameters is given by

$$
\underline{s}=\left[s_{1}, s_{2}, s_{3}, s_{4}\right]^{T}=\left[\widehat{E}_{\| 0}, \widehat{E}_{\| 1}, E_{\perp}, G_{\|}\right]^{T} .
$$




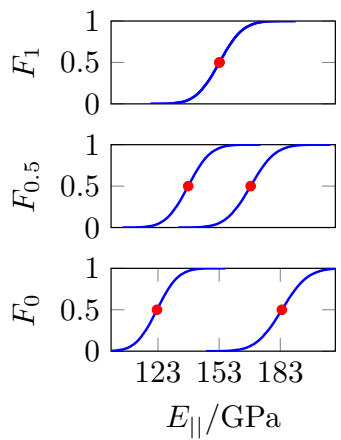

Figure 19. Hybrid fuzzy-stochastic analysis: P-boxes at $\alpha$-levels $\alpha_{1}=0, \alpha_{6}=0.5$, and $\alpha_{11}=1$ for $E_{\|}$.

The PCE in (12) for the polymorphic uncertain and stochastic parameters $E_{\|}(\omega, \underline{s})$ and $v(\omega)$ is truncated by $n_{P}=2$. The membership functions $\mu(\underline{s})$ and the density distributions for the purely fuzzy parameters $E_{\perp}(\underline{s})$ and $G_{\|}(\underline{s})$ and the purely stochastic parameter $v(\omega)$, respectively, remain unchanged according to Figures $11 \mathrm{a}, \mathrm{d}$ and 14.

According to (13), the PCEs for the (fuzzy-)random variables $E_{\|}(\omega, \underline{s})$ and $v(\omega)$ for $n_{P}=2$ are

$$
\begin{aligned}
E_{\|}(\omega, \underline{s}) & =\widehat{E}_{\| 0}(\underline{s})+\widehat{E}_{\| 1}(\underline{s}) \theta_{1}(\omega)+\widehat{E}_{\| 2}(\underline{s}) \theta_{2}(\omega), \\
v(\omega) & =\hat{v}_{0}+\hat{v}_{1} \theta_{1}(\omega)+\hat{v}_{2} \theta_{2}(\omega) .
\end{aligned}
$$

We assume, further, that $E_{\|}(\omega, \underline{s})$ and $v(\omega)$ are stochastically independent such that (56) renders

$$
\begin{aligned}
E_{\|}(\omega, \underline{s}) & =\widehat{E}_{\| 0}(\underline{s})+\widehat{E}_{\| 1}(\underline{s}) \theta_{1}(\omega), \\
v(\omega) & =\hat{v}_{0}+\hat{v}_{2} \theta_{2}(\omega),
\end{aligned}
$$

with PC coefficients

$$
\begin{aligned}
& \widehat{E}_{\| 0}(\underline{s})=\mathbb{E}\left[E_{\|}(\underline{s})\right]=s_{1}, \quad \widehat{E}_{\| 1}(\underline{s})=\operatorname{std}\left[E_{\|}(\underline{s})\right]=s_{2}, \\
& \hat{v}_{0}=\mathbb{E}[v] \quad=0.306, \quad \hat{v}_{2}=\operatorname{std}[v] \quad=0.0528 \text {. }
\end{aligned}
$$

Consequently, the corresponding input matrix in (16) of material parameter PC coefficients becomes

$$
\hat{\hat{\kappa}}(\underline{s})=\left[\begin{array}{ccc}
\widehat{E}_{\| 0}(\underline{s}) & \widehat{E}_{\| 1}(\underline{s}) & 0 \\
E_{\perp}(\underline{s}) & 0 & 0 \\
G_{\|}(\underline{s}) & 0 & 0 \\
G_{\perp} & 0 & 0 \\
\hat{v}_{0} & 0 & \hat{v}_{2}
\end{array}\right]=\left[\begin{array}{ccc}
s_{1} & s_{2} & 0 \\
s_{3} & 0 & 0 \\
s_{4} & 0 & 0 \\
3.9286 & 0 & 0 \\
0.306 & 0 & 0.0528
\end{array}\right] .
$$



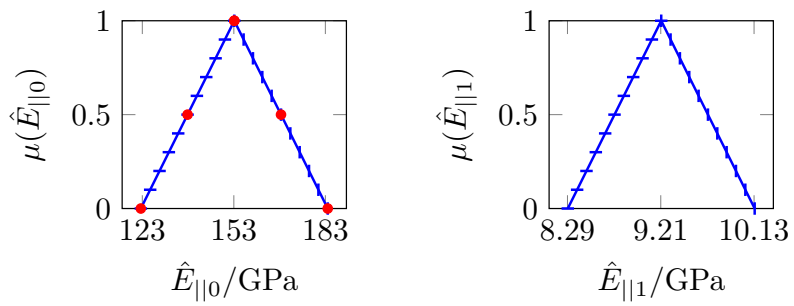

Figure 20. Hybrid fuzzy-stochastic analysis: input membership functions for (left) the zeros $\widehat{E}_{\| 0}$ and (right) first $\widehat{E}_{\| 1}$ PC coefficients of $E_{\|}$.

In this example, the corresponding artificially chosen input membership functions for $s_{1}=\mathbb{E}\left[E_{\|}(\omega, \underline{s})\right]=\widehat{E}_{\| 0}$ and $s_{2}=\operatorname{std}\left[E_{\|}(\omega, \underline{s})\right]=\widehat{E}_{\| 1}$ are shown in Figure 20. Therefore, $E_{\|}(\omega, \underline{s})$ becomes a hybrid fuzzy-stochastic variable, i.e, the CDF of $E_{\|}(\omega, \underline{s})$ is not anymore given by unique function as depicted in Figure $14 \mathrm{c}$. Instead, the CDF is given by left upper bounds $\bar{F}_{\alpha_{k}}\left(E_{\|}\right)$and right lower bounds $\underline{F}_{\alpha_{k}}\left(E_{\|}\right)$at each $\alpha$-level. Then, the P-boxes of Young's modulus $\left[\bar{F}_{\alpha_{k}}\left(E_{\|}\right), \underline{F}_{\alpha_{k}}\left(E_{\|}\right)\right]$at three $\alpha$-levels $\alpha_{1}=0, \alpha_{6}=0.5$, and $\alpha_{11}=1$ are shown in Figure 19. The red dots in Figure 19 indicate the expectation values of left upper bounds $\bar{F}_{\alpha_{k}}\left(E_{\|}\right)$and right lower bounds $\underline{F}_{\alpha_{k}}\left(E_{\|}\right)$at the three $\alpha$-levels. The same values are also shown by red dots in Figure 20, left.

The fuzzy and the stochastic analysis are combined to a hybrid fuzzy-stochastic approach. In Figure 21, the resulting output membership functions of the optimization problems in (37) for the three possible realizations of surrogate QoIs presented in (20)-(22) are illustrated. The - and I marks show that from each pair of interval bounds of the design variables $s_{i, \alpha_{k}}^{L}$ and $s_{i, \alpha_{k}}^{R}$ at each $\alpha$-level $\alpha_{k}, k=1, \ldots, 11$, in (49), the interval bounds of the QoIs $Q_{\alpha_{k}}^{\omega L}$ and $Q_{\alpha_{k}}^{\omega R}$ are obtained. Therefore, for postprocessing, e.g., calculating the stresses $\underline{\sigma}(\omega, \underline{s})$ in (38), the left and right interval bounds $s_{i, \alpha_{k}}^{L}$ and $s_{i, \alpha_{k}}^{R}$ at each $\alpha$-level $\alpha_{k}, k=1, \ldots, 11$, can be used.
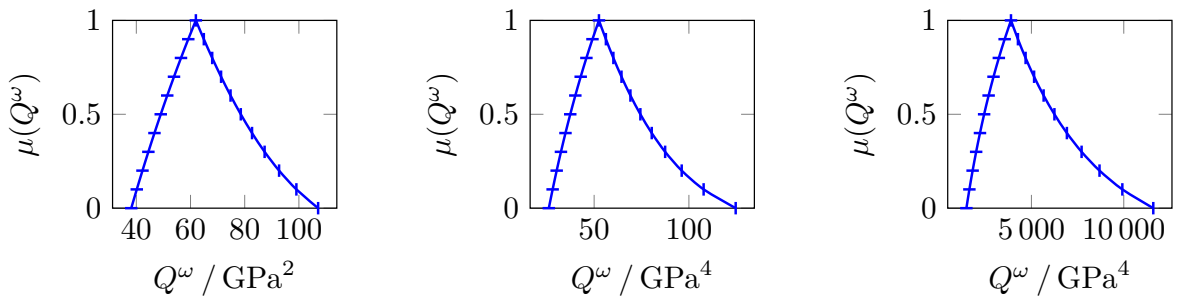

Figure 21. Hybrid fuzzy-stochastic analysis: membership function for surrogate QoIs in (20)-(22) according to QoI in (40), (left) $Q^{\omega}=\mathbb{E}[Q]$, (center) $Q^{\omega}=\operatorname{var}[Q]$, and (right) $Q^{\omega}=\mathbb{E}\left[Q^{2}\right]$. 

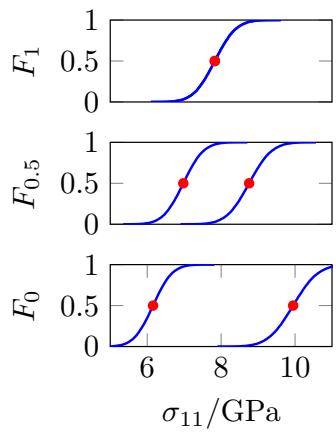
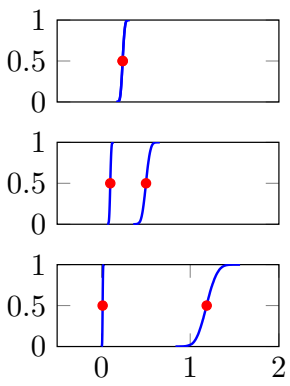

$\sigma_{22} / \mathrm{GPa}$
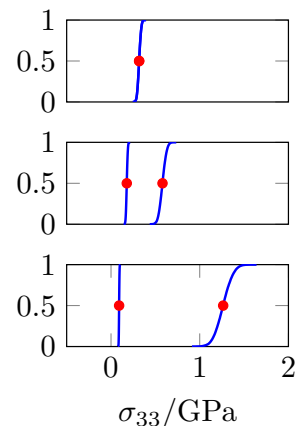

Figure 22. Hybrid fuzzy-stochastic analysis: P-boxes at $\alpha$-levels $\alpha_{1}=0, \alpha_{6}=0.5$ and $\alpha_{11}=1$ for normal stresses $\sigma_{11}, \sigma_{22}, \sigma_{33}$.

The purely fuzzy or deterministic shear stresses remain unchanged compared to the first example in Section 5.1. The P-boxes of the normal stresses $\sigma_{i i}(\omega, \underline{s})$, which depend on deterministic $G_{\perp}$, fuzzy $E_{\perp}(\underline{s})$ and $G_{\|}(\underline{s})$, stochastic $v(\omega)$, and hybrid fuzzy-stochastic $E_{\|}(\omega, \underline{s})$ parameters, are shown in Figure 22 for three $\alpha$ levels. In contrast to the previous example in Section 5.2, wider breadth is obtained between the left upper bounds $\bar{F}_{\alpha_{k}}\left(\sigma_{11}\right)$ and right lower bounds $\underline{F}_{\alpha_{k}}\left(\sigma_{11}\right)$ for each $\alpha$-level, whereas the bound distributions for $\sigma_{22}$ and $\sigma_{33}$ are narrow, which was to be expected because of the wider input of $E_{\|}$. In addition, the membership functions for the expectation values and the variances of $\sigma_{11}, \sigma_{22}$, and $\sigma_{33}$ are given in Figure 23. The sensitivity of the design variables $\underline{s}$ can be clearly seen in

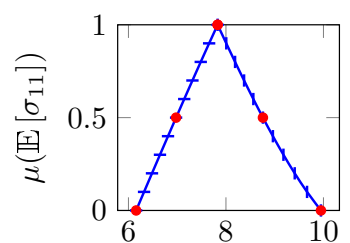

$\mathbb{E}\left[\sigma_{11}\right] / \mathrm{GPa}$

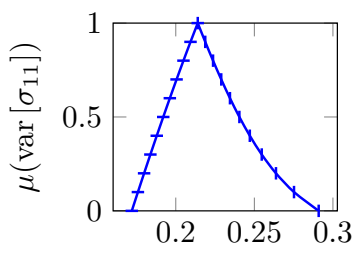

$\operatorname{var}\left[\sigma_{11}\right] / \mathrm{GPa}^{2}$

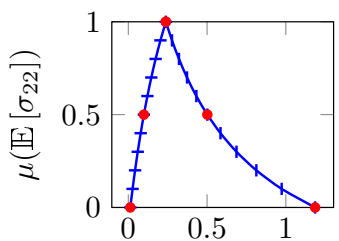

$\mathbb{E}\left[\sigma_{22}\right] / \mathrm{GPa}$

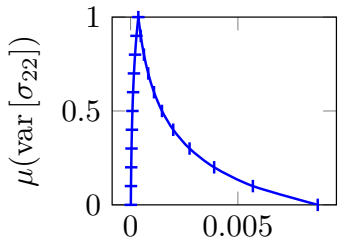

$\operatorname{var}\left[\sigma_{22}\right] / \mathrm{GPa}^{2}$

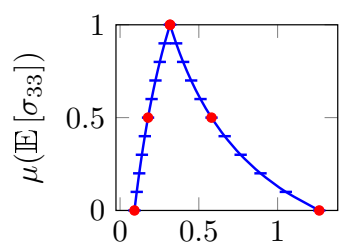

$\mathbb{E}\left[\sigma_{33}\right] / \mathrm{GPa}$

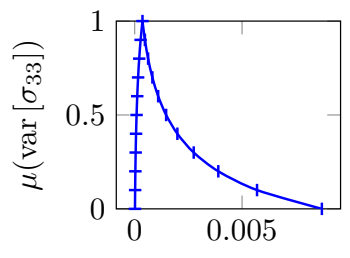

$\operatorname{var}\left[\sigma_{33}\right] / \mathrm{GPa}^{2}$

Figure 23. Hybrid fuzzy-stochastic analysis: output membership functions for expectation values (top row) and variances (bottom row) of normal stresses. 
both figures. Higher values of $\underline{s}$ result in a larger increase in statistical moments than for smaller values of $s$.

\section{Conclusion and outlook}

The objective of this article is to consider polymorphic uncertain material parameters, for unidirectional FRP, in a hybrid fuzzy-stochastic transversely isotropic elastic model. For this purpose, a state and a target problem is formulated as (fuzzy) stochastic equations that take into account PC expanded (fuzzy) random variables, where the PC coefficients are interpreted as fuzzy design variables. To this end, a stochastic Galerkin projection is applied to reduce the (fuzzy) stochastic equation into a system of (fuzzy) equations. Hence, the target problem or the QoI depends only on (fuzzy) PC coefficients. The fuzzy analysis, including $\alpha$-level optimization, is used to get representative membership functions for QoIs. Since the QoIs may be random variables, surrogate QoIs based on empirical moments are used to perform the fuzzy optimization. The material parameters for the proposed model are determined based on homogeneous experiments of tensile specimens with different fiber orientations. This is followed by statistic evaluations of material parameters. These results are used for the generation of stochastic distributions or fuzzy membership functions, respectively, and applied as input quantities for the numerical analysis. Representative examples, for fuzzy analysis, fuzzy-stochastic analysis, and hybrid fuzzy-stochastic analysis demonstrate the versatility of the proposed model.

In the future, inhomogeneous experiments will be carried out for validation and compared with the proposed hybrid fuzzy-stochastic transversely isotropic elastic model of unidirectional FRP. Furthermore, a multiscale model should be developed, which takes into account a polymorphic uncertain homogenization method, where the composite material may exhibit polymorphic uncertainties in the constituent material properties.

\section{Acknowledgements}

The support of the research in this work by "DFG Schwerpunktprogramm SPP 1886: Polymorphic uncertainty modeling for the numerical design of structures" is gratefully acknowledged.

\section{References}

[Acharjee 2006] S. Acharjee, Stochastic and deterministic techniques for computational design of deformation process, Ph.D. thesis, Cornell University, 2006.

[Acharjee and Zabaras 2006] S. Acharjee and N. Zabaras, "Uncertainty propagation in finite deformations: a spectral stochastic Lagrangian approach", Comput. Methods Appl. Mech. Engrg. 195:1922 (2006), 2289-2312. 
[Anders and Hori 1999] M. Anders and M. Hori, "Stochastic finite element method for elasto-plastic body", Internat. J. Numer. Methods Engrg. 46:11 (1999), 1897-1916.

[Beer 2009] M. Beer, "Fuzzy probability theory", pp. 4047-4059 in Encyclopedia of complexity and systems science, edited by R. A. Meyers, Springer, 2009.

[Bertsekas 1982] D. P. Bertsekas, "Projected Newton methods for optimization problems with simple constraints", SIAM J. Control Optim. 20:2 (1982), 221-246.

[Caflisch 1998] R. E. Caflisch, "Monte Carlo and quasi-Monte Carlo methods", Acta Numer. 7 (1998), 1-49.

[Caylak et al. 2018] I. Caylak, E. Penner, A. Dridger, and R. Mahnken, "Stochastic hyperelastic modeling considering dependency of material parameters", Comput. Mech. 62:6 (2018), 1273-1285.

[Dempster 1967] A. P. Dempster, "Upper and lower probabilities induced by a multivalued mapping”, Ann. Math. Statist. 38 (1967), 325-339.

[Dubois and Prade 2012] D. Dubois and H. Prade, "Possibility theory", pp. 2240-2252 in Computational complexity, edited by R. A. Meyers, Springer, 2012.

[Ferson et al. 2003] S. Ferson, V. Kreinovich, L. Grinzburg, D. Myers, and K. Sentz, "Constructing probability boxes and Dempster-Shafer structures", tech. report SAND2002-4015, Sandia National Laboratories, 2003, Available at https://www.osti.gov/biblio/1427258.

[Ghanem and Spanos 1991] R. G. Ghanem and P. D. Spanos, Stochastic finite elements: a spectral approach, Springer, 1991.

[Graf et al. 2015] W. Graf, M. Götz, and M. Kaliske, "Analysis of dynamical processes under consideration of polymorphic uncertainty”, Struct. Safety 52:B (2015), 194-201.

[Gudder 1998] S. Gudder, "Fuzzy probability theory", Demonstratio Math. 31:1 (1998), 235-254.

[Hurtado and Barbat 1998] J. E. Hurtado and A. H. Barbat, "Monte Carlo techniques in computational stochastic mechanics", Arch. Comput. Methods Engrg. 5:1 (1998), 3-29.

[Jahani et al. 2014] E. Jahani, R. L. Muhanna, M. A. Shayanfar, and M. A. Barkhordari, "Reliability assessment with fuzzy random variables using interval Monte Carlo simulation", Comput.-Aid. Civ. Infrastruct. Eng. 29:3 (2014), 208-220.

[Jardak et al. 2002] M. Jardak, C.-H. Su, and G. E. Karniadakis, "Spectral polynomial chaos solutions of the stochastic advection equation", J. Sci. Comput. 17:1-4 (2002), 319-338.

[Keese 2004] A. Keese, Numerical solution of systems with stochastic uncertainties: a general purpose framework for stochastic finite elements, Ph.D. thesis, Technische Universität Braunschweig, 2004, Available at https://publikationsserver.tu-braunschweig.de/receive/dbbs_mods_00001595.

[Le Maître and Knio 2010] O. P. Le Maître and O. M. Knio, Spectral methods for uncertainty quantification: with applications to computational fluid dynamics, Springer, 2010.

[Le Maître et al. 2001] O. P. Le Maître, O. M. Knio, H. N. Najm, and R. G. Ghanem, "A stochastic projection method for fluid flow, I: Basic formulation”, J. Comput. Phys. 173:2 (2001), 481-511.

[Le Maître et al. 2002] O. P. Le Maître, M. T. Reagan, H. N. Najm, R. G. Ghanem, and O. M. Knio, "A stochastic projection method for fluid flow, II: Random process", J. Comput. Phys. 181:1 (2002), 9-44.

[Mahnken 2017] R. Mahnken, "A variational formulation for fuzzy analysis in continuum mechanics”, Math. Mech. Complex Syst. 5:3-4 (2017), 261-298.

[Möller and Beer 2004] B. Möller and M. Beer, Fuzzy randomness: uncertainty in civil engineering and computational mechanics, Springer, 2004.

[Motamed 2017] M. Motamed, "Fuzzy-stochastic partial differential equations", preprint, 2017. arXiv 
[Nocedal and Wright 1999] J. Nocedal and S. J. Wright, Numerical optimization, Springer, 1999.

[Reuter et al. 2012] U. Reuter, J.-U. Sickert, W. Graf, and M. Kaliske, "Modeling and processing of uncertainty in civil engineering by means of fuzzy randomness", pp. 291-306 in Managing safety of heterogeneous systems, edited by Y. Ermoliev et al., Lecture Notes in Economics and Mathematical Systems 658, Springer, 2012.

[Rosić 2013] B. Rosić, "Variational formulations and functional approximation algorithms in stochastic plasticity of materials", in 2nd ECCOMAS Young Investigators Conference (Bordeaux, 2013), ECCOMAS, Barcelona, 2013.

[Soden et al. 1998] P. D. Soden, M. J. Hinton, and A. S. Kaddour, "Lamina properties, lay-up configurations and loading conditions for a range of fibre-reinforced composite laminates", Compos. Sci. Tech. 58:7 (1998), 1011-1022.

[Sullivan 2015] T. J. Sullivan, Introduction to uncertainty quantification, Texts in Applied Mathematics 63, Springer, 2015.

[Walley 1991] P. Walley, Statistical reasoning with imprecise probabilities, Monographs on Statistics and Applied Probability 42, Chapman and Hall, London, 1991.

[Wang and Zhang 1992] G. Y. Wang and Y. Zhang, "The theory of fuzzy stochastic processes", Fuzzy Sets and Systems 51:2 (1992), 161-178.

[Xiu and Karniadakis 2002] D. Xiu and G. E. Karniadakis, "The Wiener-Askey polynomial chaos for stochastic differential equations”, SIAM J. Sci. Comput. 24:2 (2002), 619-644.

[Xiu et al. 2001] D. Xiu, C.-H. Lucor, Didier Su, and G. E. Karniadakis, "Stochastic modeling of flow-structure interactions using generalized polynomial chaos", J. Fluid. Eng. 124:1 (2001), $51-59$.

[Zadeh 1965] L. A. Zadeh, "Fuzzy sets", Information and Control 8 (1965), 338-353.

Received 6 Sep 2018. Revised 9 Jan 2019. Accepted 1 Mar 2019.

EDUARD PENNER: penner@ltm.upb.de

Faculty of Mechanical Engineering, Paderborn University, Paderborn, Germany

ISMAIL CAYLAK: caylak@ltm.upb.de

Faculty of Mechanical Engineering, Paderborn University, Paderborn, Germany

ALEX DRIDGER: dridger@ltm.upb.de

Faculty of Mechanical Engineering, Paderborn University, Paderborn, Germany

ROLF MAHNKEN: rolf.mahnken@ltm.uni-paderborn.de

Faculty of Mechanical Engineering, Paderborn University, Paderborn, Germany 
EDITORIAL BOARD

ANTONIO CARCATERRA

ERIC A. CARLEN

FRANCESCO DELL'ISOLA

RAFFAELE ESPOSITO

ALBERT FANNJIANG

Gilles A. FrancFort

Pierangelo Marcati

JEAN-JACQUES MARIGO

PETER A. MARKOWICH

MARTIN OSTOJA-STARZEWSKI

PIERRE SEPPECHER

DAVID J. STEIGMANN

PAUL STEINMANN

PierRe M. SuQueT

MANAGING EDITORS

MICOL AMAR

ANGELA MADEO

MARTIN OSTOJA-STARZEWSKI

\section{ADVISORY BOARD}

ADNAN AKAY

Holm AltenbaCH

MICOL AMAR

HARM ASKES

TEODOR ATANACKOVIĆ

VICTOR BERDICHEVSKY

GuY BouchitTÉ

ANDREA BRAIDES

ROBERTO CAMASSA

MAURO CARFORE

ERIC DARVE

FELIX DARVE

ANNA DE MASI

Gianpietro Del Piero

Emmanuele Di Benedetto

VICTOR A. EREMEYEV

BERNOLD FIEDLER

IRENE M. GAMBA

DAVID Y. GAO

SERGEy GaVRILYUK

Timothy J. HeAley

DOMINIQUE JEULIN

ROgER E. KHAYAT

CORRADO LATTANZIO

ROBERT P. LIPTON

ANGELO LUONGO

ANGEla MADEO

JUAN J. MANFREDI

CARlo MARCHIORO

ANIL MISRA

ROBERTO NATALINI

PATRIZIO NEFF

Thomas J. Pence

ANDREY PIATNITSKI

ERRICO PRESUTtI

MARIO PUlVIRENTI

Lucio Russo

Miguel A. F. SANJUAN

PATRICK SElvaduraI

MIROSLAV ŠILHAVÝ

GUIDO SWEERS

ANTOINETTE TORDESILLAS

LEV TRUSKINOVSKY

JUAN J. L. VELÁZQUEZ

VINCENZO VESPRI

ANGELO VULPIANI msp.org/memocs

Università di Roma "La Sapienza", Italia

Rutgers University, USA

(CO-CHAIR) Università di Roma "La Sapienza", Italia

(TREASURER) Università dell'Aquila, Italia

University of California at Davis, USA

(CO-CHAIR) Université Paris-Nord, France

Università dell' Aquila, Italy

École Polytechnique, France

DAMTP Cambridge, UK, and University of Vienna, Austria

(CHAIR MANAGING EDITOR) Univ. of Illinois at Urbana-Champaign, USA

Université du Sud Toulon-Var, France

University of California at Berkeley, USA

Universität Erlangen-Nürnberg, Germany

LMA CNRS Marseille, France

Università di Roma "La Sapienza", Italia

Université de Lyon-INSA (Institut National des Sciences Appliquées), France (CHAIR MANAGING EDITOR) Univ. of Illinois at Urbana-Champaign, USA

Carnegie Mellon University, USA, and Bilkent University, Turkey

Otto-von-Guericke-Universität Magdeburg, Germany

Università di Roma "La Sapienza”, Italia

University of Sheffield, UK

University of Novi Sad, Serbia

Wayne State University, USA

Université du Sud Toulon-Var, France

Università di Roma Tor Vergata, Italia

University of North Carolina at Chapel Hill, USA

Università di Pavia, Italia

Stanford University, USA

Institut Polytechnique de Grenoble, France

Università dell' Aquila, Italia

Università di Ferrara and International Research Center MEMOCS, Italia

Vanderbilt University, USA

Gdansk University of Technology, Poland

Freie Universität Berlin, Germany

University of Texas at Austin, USA

Federation University and Australian National University, Australia

Université Aix-Marseille, France

Cornell University, USA

École des Mines, France

University of Western Ontario, Canada

Università dell' Aquila, Italy

Louisiana State University, USA

Università dell'Aquila, Italia

Université de Lyon-INSA (Institut National des Sciences Appliquées), France University of Pittsburgh, USA

Università di Roma "La Sapienza", Italia

University of Kansas, USA

Istituto per le Applicazioni del Calcolo "M. Picone", Italy

Universität Duisburg-Essen, Germany

Michigan State University, USA

Narvik University College, Norway, Russia

Università di Roma Tor Vergata, Italy

Università di Roma “La Sapienza”, Italia

Università di Roma “Tor Vergata", Italia

Universidad Rey Juan Carlos, Madrid, Spain

McGill University, Canada

Academy of Sciences of the Czech Republic

Universität zu Köln, Germany

University of Melbourne, Australia

École Polytechnique, France

Bonn University, Germany

Università di Firenze, Italia

Università di Roma La Sapienza, Italia

MEMOCS (ISSN 2325-3444 electronic, 2326-7186 printed) is a journal of the International Research Center for the Mathematics and Mechanics of Complex Systems at the Università dell'Aquila, Italy.

Cover image: "Tangle” by $\odot$ John Horigan; produced using the Context Free program (contextfreeart.org).

PUBLISHED BY

7 mathematical sciences publishers

nonprofit scientific publishing

http://msp.org/

(C) 2019 Mathematical Sciences Publishers 
Mathematics and Mechanics of Complex Systems vol. 7 no. 2

A polynomial chaos expanded hybrid fuzzy-stochastic model for transversely fiber reinforced plastics

Eduard Penner, Ismail Caylak, Alex Dridger and Rolf Mahnken

Dynamic boundary conditions for membranes whose surface energy depends on the mean and Gaussian curvatures

Sergey Gavrilyuk and Henri Gouin Energy-based trajectory tracking and vibration control for multilink highly flexible manipulators

Ivan Giorgio and Dionisio Del Vescovo

A model of the proppant flowback: setup of the theoretical framework

Ksenia P. Frolova, Polina M. Grigoreva, Konstantin E.

Lezhnev and Grigoriy V. Paderin

The object detection by autonomous apparatus as a solution of the 189 Buffon needle problem

Mikhail A. Guzev, Gurami S. Tsitsiashvili and Marina A.

Osipova

MEMOCS is a journal of the International Research Center for the Mathematics and Mechanics of Complex Systems at the Università dell' Aquila, Italy.

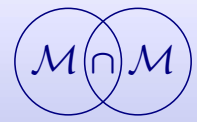

